ARTICLE

\title{
Efflux pump activity potentiates the evolution of antibiotic resistance across $S$. aureus isolates
}

\author{
Andrei Papkou (1) ${ }^{1,2,4 凶}$, Jessica Hedge (iD) ${ }^{1,4}$, Natalia Kapel ${ }^{1}$, Bernadette Young (i) ${ }^{3}$ \& R. Craig MacLean (1) ${ }^{1 凶}$
}

The rise of antibiotic resistance in many bacterial pathogens has been driven by the spread of a few successful strains, suggesting that some bacteria are genetically pre-disposed to evolving resistance. Here, we test this hypothesis by challenging a diverse set of 222 isolates of Staphylococcus aureus with the antibiotic ciprofloxacin in a large-scale evolution experiment. We find that a single efflux pump, nor $A$, causes widespread variation in evolvability across isolates. Elevated norA expression potentiates evolution by increasing the fitness benefit provided by DNA topoisomerase mutations under ciprofloxacin treatment. Amplification of norA provides a further mechanism of rapid evolution in isolates from the CC398 lineage. Crucially, chemical inhibition of NorA effectively prevents the evolution of resistance in all isolates. Our study shows that pre-existing genetic diversity plays a key role in shaping resistance evolution, and it may be possible to predict which strains are likely to evolve resistance and to optimize inhibitor use to prevent this outcome.

\footnotetext{
${ }^{1}$ Department of Zoology, University of Oxford, 11a Mansfield Road, Oxford OX1 3PS, UK. ${ }^{2}$ Department of Evolutionary Biology and Environmental Studies, University of Zurich, Winterthurerstrasse 190, Zurich CH-8057, Switzerland. ${ }^{3}$ Nuffield Department of Clinical Medicine, John Radcliffe Hospital, University of Oxford, Oxford OX3 9DU, UK. ${ }^{4}$ These authors contributed equally: Andrei Papkou, Jessica Hedge. ${ }^{{ }}$email: andrei.papkou@uzh.ch; craig.maclean@zoo.ox.ac.uk
} 
nfections caused by antibiotic resistant bacteria are currently estimated to cause $\sim 700,000$ deaths per year, and this mortality rate is predicted to increase to 10 million per year by $2050^{1}$. Given this threat, resistance has been identified as one of the most important challenges to human health by a wide variety of national and international bodies, including the WHO, the G8 and the IMF. To solve this crisis, we require new antimicrobials to treat infections caused by resistant pathogens and new approaches to predict and prevent the spread of resistance in pathogen populations $^{2-4}$.

The increase in antibiotic resistance in many pathogens has been driven by the spread of a relatively small number of very successful antibiotic resistant lineages ${ }^{5-10}$. One explanation for this pattern is that these successful lineages are simply those that, by chance, successfully acquire rare antibiotic resistance genes by mutation or horizontal gene transfer ${ }^{11,12}$. Alternatively, it is possible that some strains of bacteria are more likely to evolve resistance than others, for example because they have an elevated mutation rate $^{13}$, or because they carry 'potentiator' genes that open up new genetic paths to evolving resistance ${ }^{14,15}$. If so, it may be possible to identify strains of bacteria that are at high risk of evolving resistance to antibiotics, and to change antibiotic usage to prevent this outcome and associated treatment failure ${ }^{16}$.

In this paper, we investigate genomic drivers of evolvability using comparative experimental evolution. In this approach, many bacterial 'parental' strains are challenged with a common selective pressure using controlled and highly replicated in vitro evolution experiments. Following the trajectory of replicate selection lines after antibiotic exposure makes it possible to estimate the evolvability of each strain, and downstream phenotypic and genomic analyses are used to characterize evolved populations ${ }^{14,17}$. This approach makes it possible to address three separate questions: (i) Does the ability to evolve antibiotic resistance differ between bacterial strains? (ii) What are the underlying mechanisms of resistance evolution? (iii) How does genome content determine the rate and mechanisms of resistance evolution?

We applied this approach at a very large scale by challenging a diverse collection of 222 parental strains of Staphylococcus aureus with ciprofloxacin using a highly replicated selection experiment. Parental strains were sampled from a large collection of nasal carriage and bacteraemia isolates of $S$. aureus collected at hospitals in Oxford and Brighton, $\mathrm{UK}^{18}$, and were chosen to capture a diverse set of clonal-complexes of humanassociated $S$. aureus. We chose to focus on ciprofloxacin because the evolution of ciprofloxacin resistance has played a key role in the success of MRSA lineages ${ }^{19}$, and has been associated with poor clinical outcomes for patients infected with CC22 S. aureus ${ }^{20}$.

\section{Results}

Evolution of ciprofloxacin resistance across S. aureus. We serially passaged 12 replicate populations of 222 parental strains of $S$. aureus into fresh culture medium supplemented with the clinical breakpoint concentration of ciprofloxacin $\left(1 \mathrm{mgl}^{-1}\right)$ for 5 days and recorded bacterial density over time (Fig. 1a, b and Supplementary Fig. 1). All of the parental strains were ciprofloxacin sensitive according to EUCAST clinical breakpoint $(\mathrm{MIC}<$ $1 \mathrm{mgl}^{-1}$ ) and we initiated our populations with an inoculum small enough $\left(\sim 3 \times 10^{5}\right.$ cells $)$ to virtually guarantee that no preexisting resistance mutations were present at the outset of the experiment $\left(\mathrm{cip}^{\mathrm{R}}\right.$ mutation rate $5.1 \times 10^{-9}$ per cell division, $95 \%$ $\left.\mathrm{CI}=\left[3.7 \times 10^{-9}, 6.7 \times 10^{-9}\right]\right)$. Conventional antibiotic treatment strategies rely on using large doses of antibiotics to clear pathogen populations, and this simple experimental design seeks to capture this by creating a scenario in which populations must either evolve resistance or face extinction.

Bacterial population density declined at the start of the experiment due to the bactericidal effects of ciprofloxacin and the population bottlenecking imposed by serially passaging cultures. However, population density eventually recovered in a subset $(1075$ of $2664 ; 40 \%)$ of cultures, suggesting that these populations had expanded due to the de novo evolution of ciprofloxacin resistance during our experiment. To test this idea, we measured the ciprofloxacin MIC for 83 populations that recovered, each derived from a different parental strain (Supplementary Fig. 2). All evolved populations had an MIC > $1 \mathrm{mgl}^{-1}$, demonstrating that population recovery was driven by the evolution of resistance. Therefore, we measured the evolvability of ciprofloxacin resistance in the parental strains as the fraction of populations that survived until the end of the selection experiment. Our selection experiment revealed striking variation in evolvability across $S$. aureus; for example, resistance always evolved in 24 strains, whereas resistance never evolved in 39 strains (Supplementary Fig. 3a).

All else being equal, strains with a higher initial resistance to ciprofloxacin should have a greater opportunity to evolve resistance to a fixed dose of ciprofloxacin. For example, populations of strains with higher initial resistance should decline more slowly under ciprofloxacin treatment, allowing more opportunity for the emergence of novel ciprofloxacin resistance mutations ${ }^{21}$. To test this hypothesis, we measured the growth of the parental strains across a fine gradient of ciprofloxacin concentrations, and used growth data to calculate a ciprofloxacin $\mathrm{MIC}$ and $\mathrm{IC}_{50}$ for each strain. Although the parental strains were all ciprofloxacin sensitive with respect to the EUCAST clinical breakpoint (i.e. MIC $\leq 1 \mathrm{mgl}^{-1}$ ), we found subtle and quantitative variation in initial resistance between the parental strainsfor the rest of the manuscript we refer to this variation as the intrinsic resistance of the parental strains (Supplementary Fig. 4).

Evolvability increased with intrinsic ciprofloxacin resistance, and intrinsic resistance accounts for $27 \%$ of the variation in evolvability between parental strains (Pearson's correlation $r=$ $0.55, N=222, P=2 \mathrm{e}-18$; Supplementary Table 1$)$. One interesting insight from this analysis is that small differences in intrinsic resistance between strains were associated with large differences in evolvability. For example, using a logistic regression we estimated that a strain with $\mathrm{IC}_{50}=0.12 \mathrm{mg} \mathrm{l}^{-1}$ (median across all strains) would have a 0.36 probability of evolving resistance. Decreasing or increasing the $\mathrm{IC}_{50}$ by only $0.1 \mathrm{mg} \mathrm{l}^{-1}$ (equivalent to $1 / 10$ of the selection dose) would change the predicted evolvability to 0.10 or 0.72 , correspondingly (Supplementary Fig. 3b, Supplementary Table 1, 2). In contrast, there was no correlation between the resistance (i.e. $\mathrm{IC}_{50}$ ) of evolved populations and their parental strains, suggesting that the mechanisms of high level resistance that evolved during the selection experiment were independent of the intrinsic resistance of the parental strains (Pearson's correlation $r=-0.03, N=83, P=$ 0.7989).

It is also possible that some strains of $S$. aureus are genetically pre-disposed to evolving resistance, for example because they have a high mutation rate ${ }^{13,22}$, or alternative mechanisms for evolving resistance ${ }^{14}$. To test if strains belonging to different lineages of $S$. aureus evolved resistance at different rates, we grouped all parental strains into 14 non-overlapping clusters based on genetic distance estimated from whole genome data (see "Methods") 23,24. We chose to focus on these clusters, rather than pre-defined clonal complexes, because some clonal complexes, such as CC1 and CC8 were very prevalent in our parental strains, whereas other clonal complexes were represented by only few strains, making it difficult to accurately quantify variability within 


\section{a}

S. aureus strains, $N=222$

10,000 SNPs

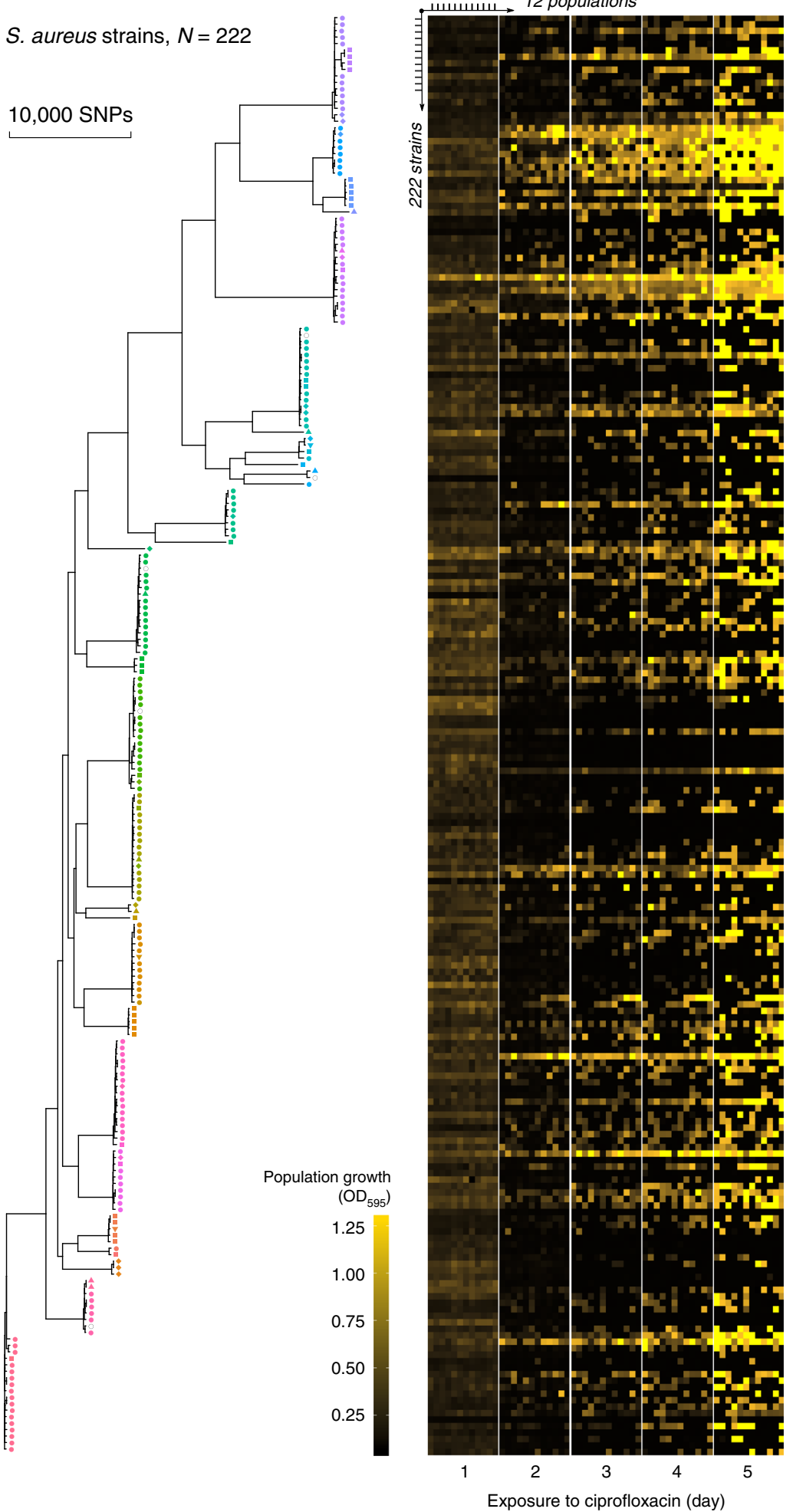

b

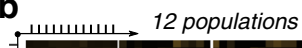

C
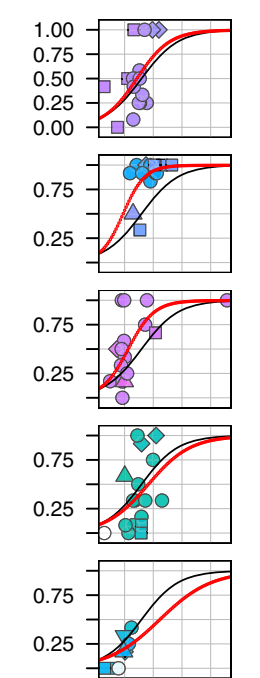

$8 \begin{aligned} & 121 \\ & 3449\end{aligned}$

$\nabla 1693$

130
ㅁ 425

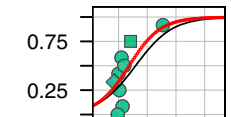

․ 3424

$\diamond 22$

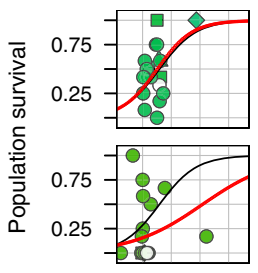

$\begin{array}{ll}\square & 88 \\ 0 & 5 \\ \triangle & 146 \\ \nabla & 101\end{array}$
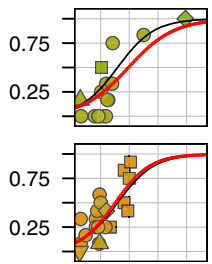



\begin{tabular}{ll} 
& 45 \\
ㅁ 3447 \\
\hdashline & 54
\end{tabular}

854

$\begin{array}{r}\nabla 50 \\ \circ 59 \\ \hline\end{array}$

3453
$\checkmark 1224$

\section{95
3449
1693
130
425
182}
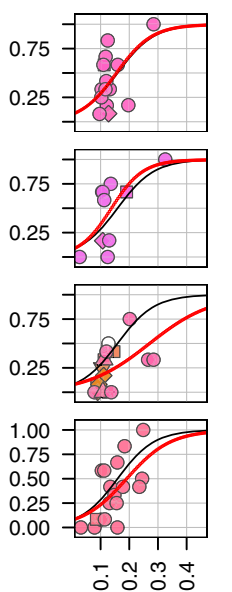

$\begin{array}{ll} & 3462 \\ \diamond & 3451 \\ 0 & 8\end{array}$

$\begin{array}{ll} & 15 \\ \text { 口 } 2434\end{array}$

2434
$8 \quad 1535$
2255

97
$0 \quad 25$

\begin{tabular}{l}
125 \\
$\times 26$ \\
\hline
\end{tabular}

3460
$\nabla 73$

O 20
$\square \quad 1281$

망 3434

$\diamond 3446$

$\begin{array}{ll} & 188 \\ \circ & 1533 \\ \diamond & 3469\end{array}$

\section{2
$\therefore \quad 3448$
$\nabla \quad 789$ \\ $\nabla 789$

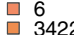 \\ $\diamond 109$}

\begin{tabular}{l}
$\circ 12$ \\
\hline \\
\hline
\end{tabular} 439

Intrinsic resistance, $\mathrm{IC}_{50}\left(\mathrm{mg} \mathrm{I}^{-1}\right)$ and between clonal complexes (Supplementary Tables 3 and 4). This sampling bias was not deliberate; rather, our pool of parental strains was skewed towards dominant ciprofloxacin-sensitive strains found in the UK.

Evolvability varied significantly between clusters, and amongcluster variation accounted for an additional $16.6 \%$ of the variation in evolvability (Fig. 1c, Supplementary Tables 1-3). This effect was largely driven by the fact that clusters with high levels of intrinsic resistance also had high evolvability (Supplementary Fig. 5). However, we found significant variation in evolvability between clusters after correcting for intrinsic resistance, implying that some strains of $S$. aureus have elevated evolvability that cannot be explained simply by high intrinsic resistance (likelihood ratio test $\chi^{2}=187.13$, d.f. $=13, P=5.94 \mathrm{e}$ -33 ). For example, strains from cluster 10, which is made up CC398, evolved resistance at a much higher rate than would have 
Fig. 1 Experimental evolution of ciprofloxacin resistance. a Maximum-likelihood phylogeny of S. aureus strains included in this study. The tree was constructed using a whole-genome alignment of 222 strains mapped to the $S$. aureus MRSA252 reference genome and corrected for recombination. b Population growth during experimental evolution. Five heatmaps show optical density $(\lambda=595)$ of bacterial populations at the end of each transfer. The optical density varies from low (no growth, $\mathrm{OD}_{595}<0.08$, black) to high density (high growth, $\mathrm{OD}_{595}>1$, bright yellow). In each heatmap, the columns correspond to 12 replicate populations, and the rows correspond to 222 strains. c The correlation of population survival and intrinsic resistance in 14 phylogenetic clusters. Each data point represents one strain where population survival is the proportion of replicate populations that evolved resistance, and intrinsic resistance is $\mathrm{IC}_{50}$ (half growth inhibition dose) of a parental strain. Colour and shape for each strain indicate MLST (multilocus sequence type). An open circle is shown when MLST could not be determined. 222 strains were assigned to 14 phylogenetic clusters based on a genetic distance matrix obtained from the phylogenetic tree in (a). The black curves show a model fit for the logistic regression between survival and intrinsic resistance across all 222 strains (the same for all clusters, GLM: $\chi^{2}=393.93$, d.f. $=1$, residual d.f. $=220, p<2.2 \mathrm{e}-16$ ), and the red curves show cluster-specific effects. Post hoc tests for cluster-specific effects are shown in Supplementary Table 6.

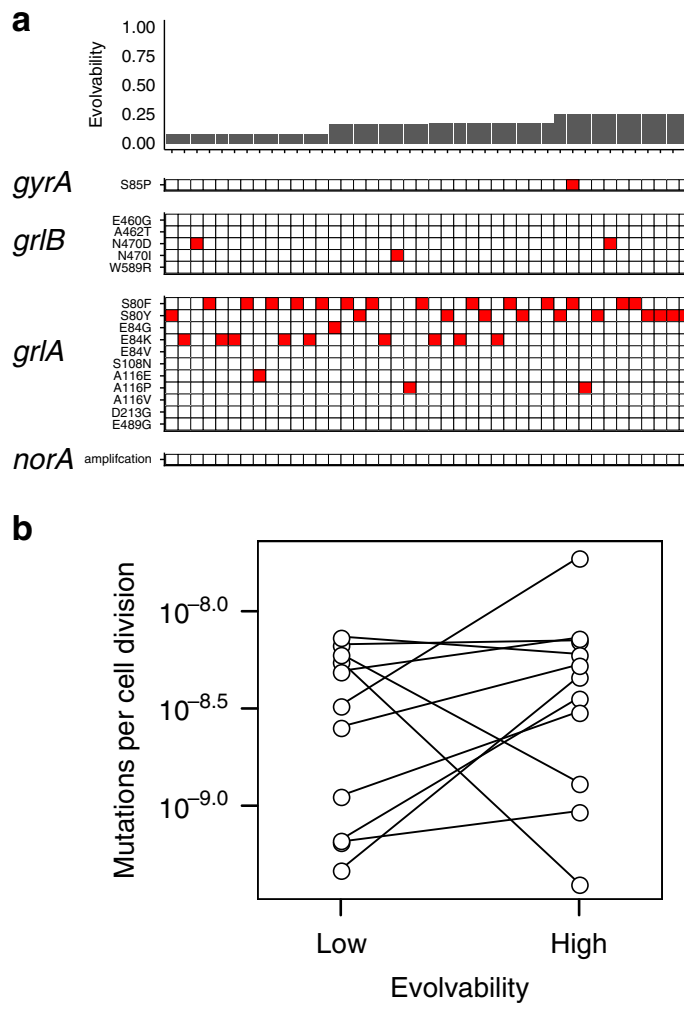

Fig. 2 Genomic basis of evolved ciprofloxacin resistance. a Resistance mutations in the evolved S. aureus populations identified by whole-genome sequencing $(N=121)$. The panels shows the identified mutations in $g y r A$, $g r l B$ and $g r l A(r e d)$ or the amplification of norA gene (blue). The populations are ranked by the evolvability of their parental strains shown as barplots on top of the panel. $\mathbf{b}$ Mutation rate in high and low evolvability strains. We measured the mutation rate to rifampicin resistance using a Luria-Delbruck fluctuation test. Lines in the figure connect pairs of high and low evolvability strains ( $N=$ 11 pairs). Two-sided Wilcoxon signed-rank test: $W=0.8311, P=0.8311, N=12$. c Copy number across sites spanning the 7239 bp region that is amplified in ST3535 and ST291 evolved strains. Copy number was calculated by summing the number of reads per site, normalized by the mean sequencing depth across all sites mapping to the ST291 reference genome, and smoothed using a generalized additive model. Gene annotations are shown below: yellow = ISSau1 transposases; blue $=$ norA.

been expected based on their intrinsic resistance (Supplementary Table 5, cluster 10, Supplementary Fig. 5).

Genomic basis of resistance evolution. One simple hypothesis to explain variation in evolvability between clusters is that some strains might have access to evolutionary paths to ciprofloxacin resistance that are not accessible to others ${ }^{14,15}$. To test this hypothesis, we sequenced the genome of a single evolved population from each of 121 parental strains that spanned the spectrum of evolvability.

The canonical mechanism for $S$. aureus to evolve ciprofloxacin resistance is by point mutations that alter ciprofloxacin targets, including topoisomerase IV ( $g r l A, g r l B)$ and DNA gyrase ( gyrA, gyrB $)^{25,26}$. Most $(106 / 121 ; 88 \%)$ evolved populations had a single
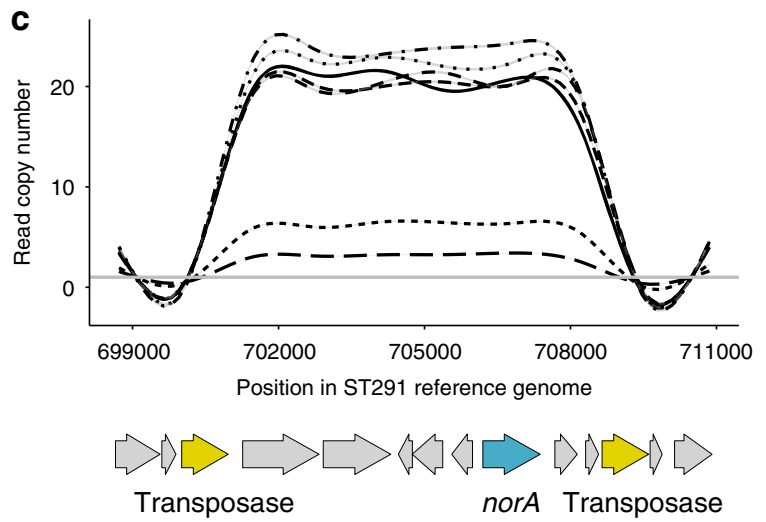
replacement mutations that alter protein function relative to what we would expect from spontaneous mutation alone ${ }^{31}$. Replacement mutations $(n=33)$ were more common than silent $(n=3)$ mutations, but the excess of replacement mutations was only marginally greater than the neutral expectation $\left(K_{\mathrm{a}} / K_{\mathrm{s}}=2.80\right.$; $N=36 ; P=0.095)$, suggesting that many non-target site mutations were neutral mutations that hitch-hiked to high frequency with adaptive resistance mutations. The key insight from this analysis is that resistance usually evolved through SNPs in welldefined ciprofloxacin targets, demonstrating a common evolutionary path to resistance in both high and low evolvability strains.

Given the key role of SNPs in resistance, variation in the underlying mutation rate might drive differences in evolvability between strains, as is the case in M. tuberculosis ${ }^{13}$. To test this hypothesis, we measured the mutation rate of pairs of high and low evolvability strains sampled across the $S$. aureus phylogeny. Mutation rate varied substantially across strains, but high evolvability was not associated with an elevated spontaneous mutation rate (Fig. 2b, two-sided Wilcoxon signed-rank test; $P=0.83, N=12$ ). Furthermore, we found no evidence of an increased substitution rate in high evolvability strains during the selection experiment (Supplementary Fig. $6, \mathrm{~W}=273.5, P=0.16$, two-sided Mann-Whitney $U$ test). Although mutation rate varies between ancestral strains, we found no evidence that high evolvability was associated with hypermutator strains.

One of the most conspicuous cases of high evolvability is the ST291 strains from clonal complex CC398. All eight strains from this group evolved resistance at a high rate, but only $1 / 8$ evolved populations had a classical resistance SNP. Instead, the evolved populations from ST291 lineage carried 3-24-fold amplifications of a $\sim 7 \mathrm{~kb}$ region of the chromosome that includes norA (Fig. 2c), an efflux pump that contributes to the intrinsic resistance of $S$. aureus towards ciprofloxacin ${ }^{32,33}$. Although the extent of norA amplification was impressive, the resistance of evolved populations with norA amplification (median $\mathrm{MIC}=8 \mathrm{mgl}^{-1} ; n=7$ ) was only marginally higher than the resistance of populations with topoisomerase substitutions (median $\mathrm{MIC}=4 \mathrm{mg} \mathrm{l}^{-1} ; \mathrm{N}=$ 69; two-sided Wilcoxon rank sum test $W=138.5, P=0.0474$ ).

The amplified region is flanked by two homologous copies of an IS30 family transposase (ISSau1), suggesting that amplification could have been caused by either transposition to different sites in the genome or tandem amplification driven by the homologous copies of ISSau1 ${ }^{34}$. To discriminate between these possibilities, we used ISmapper ${ }^{35}$ to search for new transposon insertion sites in the chromosome. We did not detect any new ISSaul insertion sites in the evolved populations, demonstrating that tandem amplification, which can occur at a much higher rate than point mutations ${ }^{36,37}$, drove the increase in nor $A$ copy number. While the ISSAul transposon is also found in a few strains outside of CC398 (Supplementary Fig. 7), we speculate that norA amplification is not observed in those strains because nor $A$ is not flanked by nearby copies of ISSAu1, as determined by re-analysing the genomes of parental strains.

Given the uniform selective pressure imposed by our experiment, we were surprised by the extent of variation in ISSaul-mediated gene amplification. All of the evolved populations with gene amplification had increased ciprofloxacin MIC, but ciprofloxacin resistance was not correlated with norA copy number (Pearson's correlation $r=-0.5733, N=7, P=0.1374$ ), suggesting that selection for resistance does not explain variation in the extent of norA gene amplification. Gene amplification is often associated with fitness costs ${ }^{37}$, suggesting that selection to minimize the cost of resistance may drive variation in gene amplification. However, we found no detectable costs of gene amplification, suggesting that selection for high growth rate did not shape variation in nor A copy number (Supplementary Fig. 8, two-sided Wilcoxon signed-rank test, $N=16, P=0.3125)$.

In summary, whole genome sequencing allowed us to uncover the mechanistic basis of evolved ciprofloxacin resistance in $(114 / 121=94 \%)$ of evolved populations. Most strains of S. aureus evolved ciprofloxacin resistance via canonical mutations in DNA topoisomerase, albeit at different rates. In contrast, strains from CC398 evolved resistance at a very high rate via a different mechanism involving amplification of the nor $A$ efflux pump gene mediated by a lineage-specific IS element. We found no evidence of parallel evolution in the remaining seven strains, suggesting that they acquired less common mechanisms of ciprofloxacin resistance (see a list of mutations in Supplementary Data 2).

Transcriptomic insights into high evolvability. Sequencing the genomes of evolved populations does not provide any obvious insights into why the rate of evolution of resistance by target alteration varied so widely among non-CC398 strains. One possible solution to this problem is that 'potentiator' genes that alter the fitness effects of topoisomerase mutations may underpin variation in evolvability in non-CC398 strains. To search for candidate potentiator genes, we sequenced the transcriptomes of 14 pairs of high and low evolvability parental strains after exposure to ciprofloxacin. These pairs of parental strains were sampled from different parts of the phylogeny by choosing pairs of strains which are closely related (based on genetic distance) yet showed a striking difference in their evolvability (survival $\geq 9 / 12$ and $\leq 1 / 12$, Supplementary Table 6 ). The goal of this paired design was to identify genes that are consistently associated with high evolvability across a range of genetic backgrounds. Gene expression was always measured after $1.5 \mathrm{~h}$ of exposure to $1 \mathrm{mgl}^{-1}$ of ciprofloxacin to match the conditions of the evolution experiment, and our transcriptome experiment did not measure basal gene expression in the absence of ciprofloxacin stress ${ }^{38}$.

In total, we identified 179 genes that were differentially expressed between high and low evolvability parental strains (Fig. 3a; Supplementary Data 3). Ciprofloxacin induces the SOS response, which provides both protection against ciprofloxacin and increased mutagenesis ${ }^{39}$, making SOS expression an obvious candidate mechanism to potentiate resistance evolution ${ }^{38}$. The SOS regulon was expressed in all strains, but there was no difference in the expression level of SOS regulated genes between high and low evolvability strains (Supplementary Table 7). Instead, overexpressed genes in high evolvability strains were enriched for metabolic functions, while genes that were overexpressed in low evolvability strains were enriched for DNA repair and nucleotide biosynthesis, which is consistent with the DNA damage caused by ciprofloxacin treatment (Supplementary Data 4). The only overexpressed gene in high evolvability strains with a known role in resistance was nor A, suggesting that this efflux pump might play a very general role in the evolution of ciprofloxacin resistance across $S$. aureus (Fig. 3b).

Testing the ability of norA to potentiate resistance evolution. To directly test the role of nor $A$ expression in evolution, we cloned norA into RN4220, a genetically tractable model strain with low evolvability. Overexpressing nor A from its native promoter marginally increased ciprofloxacin resistance, as we would expect given the established role of this pump in exporting ciprofloxacin from the cytoplasm. However, this effect was very weak, and norA overexpression was not actually sufficient to increase the MIC of RN4220 above the clinical break-point concentration of $1 \mathrm{mgl}^{-1}$ (Fig. 4a, Supplementary Table 8). To test the role of elevated nor $A$ expression in evolution, we repeated our evolution experiment by passaging 40 replicate cultures of 

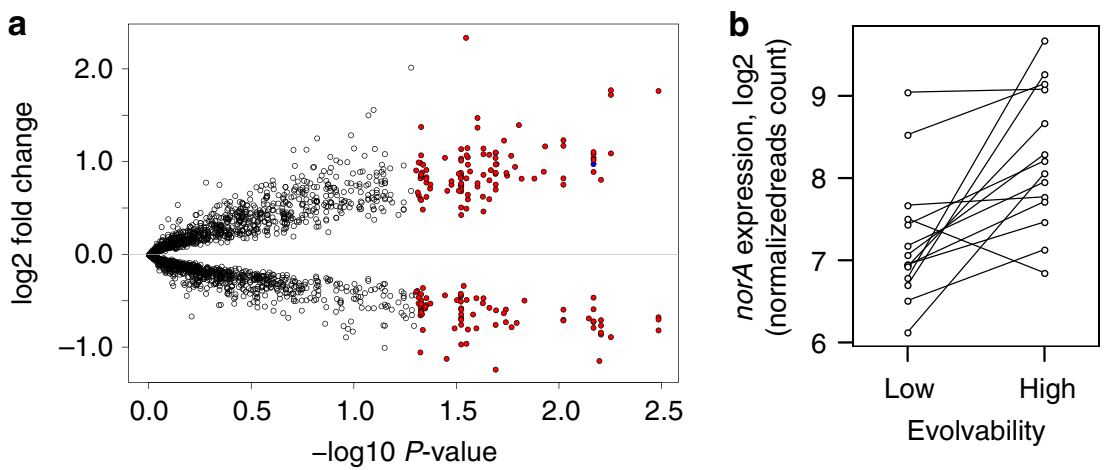

Fig. 3 Gene expression analysis. a Plotted points show the average difference (high evolvability-low evolvability) in gene expression between 14 pairs of high and low evolvability strains. Gene expression was measured after $1.5 \mathrm{~h}$ of exposure to ciprofloxacin $\left(1 \mathrm{mg} \mathrm{I}^{-1}\right)$ and each point represents a gene in the MRSA252 transcriptome (the number of genes is $n=2047)$. Significantly differentially expressed genes are coloured red $(p<0.05$, two-sided Wald tests) and norA is coloured blue. P-values were adjusted using Benjamini-Hochberg method. $\mathbf{b}$ The expression of norA in low and high evolvability strains. The read counts for the norA gene were normalised by sequencing depth. Pairs of strains are connected with a line (the number of pairs was $N=14$ ). Two-sided Wald test: $\log _{2}$ fold-change $=0.979$, standard error $=0.246, t=3.979$, adjusted $p=0.006802703$ ).

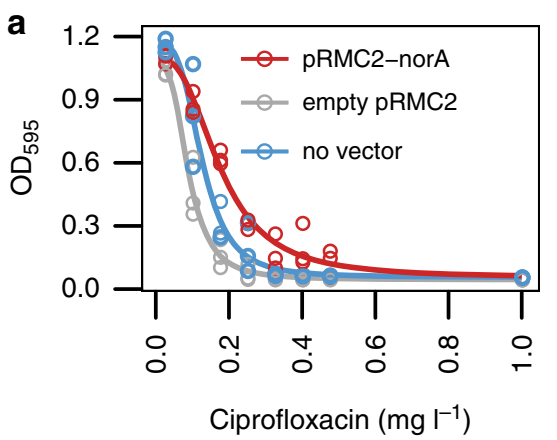

C

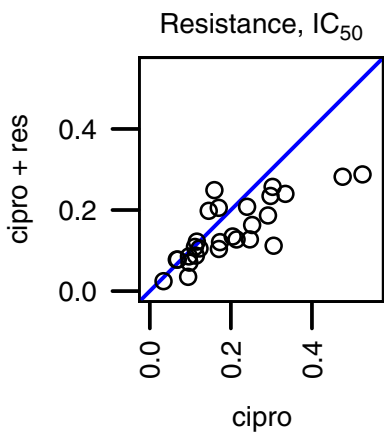

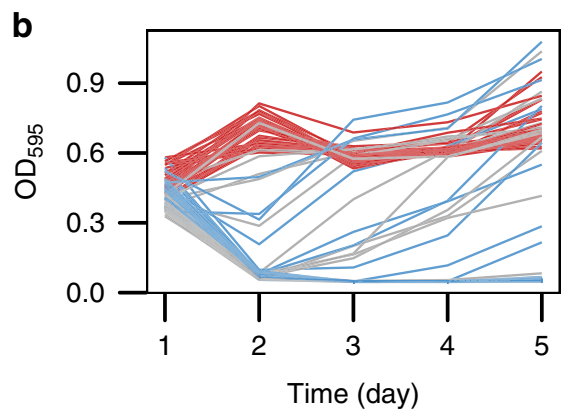

d

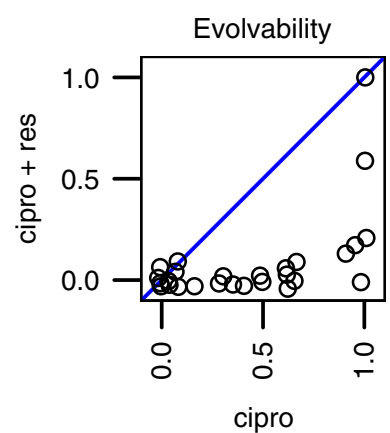

Fig. 4 The role of norA in resistance evolution. a The effect of norA overexpression on ciprofloxacin resistance. Resistance was measured in the RN4220 cells overexpressing norA from vector pRMC2 under a native promoter (red), compared to an pRMC2 empty vector control (grey) and a vector-free control (blue). $N=3$ independent cultures were used per treatment per concentration. Dose-response curves show model fit from the analysis presented in Supplementary Table 8. $\mathbf{b}$ The effect of norA overexpression on the evolution of ciprofloxacin resistance. Optical density $(\lambda=595)$ was measured for five daily transfers with $1 \mathrm{mg} \mathrm{I}^{-1}$ of ciprofloxacin in RN4220 cells overexpressing norA from the pRMC2-norA vector (red), cells with the empty pRMC2 vector (blue) and the cells without the vector (grey). $N=40$ independent cultures were used for each type of cells. Statistical analysis in shown in Supplementary Table 10. c The effect of reserpine on intrinsic resistance. Intrinsic resistance to ciprofloxacin $\left(\mathrm{IC}_{50}, \mathrm{mg} \mathrm{I}^{-1}\right)$ was determined for a representative set of 27 strains in the presence ( $y$-axis) or absence of $33 \mu \mathrm{M}$ reserpine ( $x$-axis) ( $N=5$ per dose/reserpine combination). Two-sided Wilcoxon signed-rank test: $W=308$, d.f. $=26, p=0.003$. d Evolvability was determined for the same set of 27 strains with $33 \mu M$ reserpine ( $y$-axis) or without reserpine ( $x$-axis) Evolvability was measured as the probability of population survival after 5 serial transfers at $1 \mathrm{mg} \mathrm{I}^{-1}$ of ciprofloxacin $(N=16$ replicate populations for each strain/reserpine combination). Two-sided Wilcoxon signed-rank test: $W=226, N=27, p$-value $=0.000128$.

strain RN4220 in ciprofloxacin supplemented media under basal or elevated levels of nor $A$ expression. In contrast to the subtle effect of nor $A$ overexpression on ciprofloxacin resistance, we found that nor $A$ overexpression had a dramatic effect on population survival under sustained ciprofloxacin treatment, transforming RN4220 from a low evolvability (9/40 populations) to high evolvability (40/40 populations) strain (Fig. 4b; Supplementary Tables 9, 10).

Despite the recent progress in $S$. aureus forward genetics methods ${ }^{40}$, it remains a difficult task to test the importance of norA across many $S$. aureus strains. To overcome this problem, we re-assayed the intrinsic resistance of a sub-set of our parental 
strains $(N=27)$ in the presence and absence of reserpine, a chemical inhibitor of NorA ${ }^{41}$ (Supplementary Fig. 9a, Supplementary Table 11). Reserpine treatment reduced intrinsic resistance to ciprofloxacin (Fig. 4c). Although this impact of reserpine on intrinsic resistance was evident, it translated on average into less than twofold reduction $\left(\mathrm{IC}_{50} / \mathrm{IC}_{50} \mathrm{RES}=1.31\right.$ \pm 0.47 s.d.; $\mathrm{MIC} / \mathrm{MIC}_{\mathrm{RES}}=1.52 \pm 0.49$ s.d.), with a stronger inhibition of strains with high intrinsic resistance (Spearman's correlation $\rho=0.76, p=7.4 \mathrm{e}-06, n=27)$, suggesting that this higher intrinsic resistance is at least partly based on the efflux of ciprofloxacin.

To understand the evolutionary consequence of norA expression more broadly, we repeated our evolution experiment by passaging 16 replicate cultures of these 27 strains in media containing ciprofloxacin, or both ciprofloxacin and reserpine (Supplementary Fig. 9b; Supplementary Tables 12, 13). The overall effect of reserpine was to suppress the evolution of resistance (two-sided Wilcoxon signed-rank test: $W=226$, $N=27, \quad P=0.000128)$. Reserpine had little effect on low evolvability strains, but it almost completely suppressed the evolution of ciprofloxacin resistance in high evolvability strains, including strains from CC398 (Fig. 4d). Collectively, these results show that norA expression modulates the intrinsic resistance of $S$. aureus to ciprofloxacin and potentiates the subsequent evolution of clinically relevant levels of resistance. These findings provide a simple explanation for the positive correlation between intrinsic ciprofloxacin resistance and evolvability that occurs in S. aureus (Fig. 1).

Evolutionary consequences of norA expression. The most obvious effect of norA expression is to provide protection against ciprofloxacin. This protective effect of norA could accelerate the evolution of resistance by providing populations with more time to generate resistance mutations until population extinction ${ }^{42,43}$. To test the plausibility of this mechanism, we measured the population dynamics of strain RN4220 under ciprofloxacin treatment (Fig. 5a). As expected, increased norA expression reduced the rate of population decline under ciprofloxacin treatment. However, the magnitude of this effect was marginal-norA overexpression simply delayed the onset of ciprofloxacin-induced cell mortality by $\sim 1 \mathrm{~h}$. This weak effect of norA overexpression on cell viability further highlights the fact that this efflux pump makes a small contribution to the intrinsic resistance of $S$. aureus to high doses of ciprofloxacin (i.e. $1 \mathrm{mgl}^{-1}$; Fig. 4a). Importantly, it is very difficult to reconcile this marginal effect of norA on cell viability with the massive effect of norA overexpression on evolvability (Fig. 4b), suggesting that norA expression does not accelerate evolution by increasing the rate of appearance of resistance mutations.

The appearance of a ciprofloxacin resistance mutation in a population does not guarantee that a population will successfully evolve resistance, because stochastic processes can lead to the extinction of small populations of resistant mutants, for example during population bottlenecks ${ }^{44}$. All else being equal, mutants with a high fitness upon antibiotic exposure should be more likely to successfully expand their populations and cause populations to evolve elevated resistance. To test the impact of norA expression on fitness, we measured the growth rate of RN4220 carrying key ciprofloxacin resistance SNPs under basal and elevated levels of norA expression (Supplementary Fig. 10). Elevated norA expression did not increase growth rate in wild-type RN4220, which is consistent with our previous ciprofloxacin susceptibility assays (Fig. 4a). In contrast, increased norA expression led to large increases in growth rate in ciprofloxacin resistant mutants, demonstrating positive epistasis between target alteration and antibiotic efflux (Fig. 5b).

nor A expression could increase the fitness benefit provided by resistance mutations in the presence of ciprofloxacin by reducing the fitness costs of topoisomerase substitutions and/or increasing the protective effect of topoisomerase mutations. To test this hypothesis, we measured the growth of ciprofloxacin resistant mutants under basal and elevated levels of norA expression across a range of ciprofloxacin concentrations (Fig. 5c). Elevated norA expression did not change the growth rate of resistant mutants in the absence of antibiotics (two-sided paired t-test: $t=0.42009, P$ $=0.6825, N=12$, Supplementary Fig. 10a), providing further evidence that the expression of this pump has marginal effects on fitness per se (see Fig. 4a and Supplementary Fig. 8). In contrast, we found that elevated norA expression dramatically increased the ability of grlA mutants to grow at high concentrations of ciprofloxacin (i.e. $1 \mathrm{mg} \mathrm{l}^{-1}$, two-sided paired $t$-test: $t=-18.712$, $P=1.09 \mathrm{e}-09, N=12)$. In other words, positive epistasis occurs because ciprofloxacin efflux mediated by norA and altered topoisomerase structure (i.e. grlA) interact synergistically to increase resistance to high doses of ciprofloxacin without imposing any additional fitness burden.

Identifying variants associated with high evolvability. In order to systematically search for genetic variants associated with high evolvability, we carried out a genome-wide association study (GWAS) to test for associations between core-genome SNPs and evolvability across the strains used in this study (Supplementary data 5). The GWAS analysis showed that $16.3 \%$ of variation in evolvability can be explained by genetic variability across strains (Supplementary Fig. 11). However, after controlling for multiple testing, the GWAS failed to identify any SNPs that show a significant association with high evolvability.

Given the strong association between norA and evolvability, we then focused more closely on variants that are likely to be associated with norA expression and function. All of the strains used in this study are predicted to carry a functional copy of norA (i.e. no pre-mature stop codons or frameshift mutations). In line with previous work ${ }^{45}$, we found that the norA coding sequence is polymorphic, and we identified polymorphisms in a previously characterized norA promoter region ${ }^{46-48}$. However, the $P$ values associated with these polymorphisms are 1000 times higher than the conservative significance threshold used in our GWAS analysis, suggesting that these associations are true negatives. Moreover, we failed to identify any nor $A$ variants associated with high evolvability through manual inspection of our data.

The expression of norA is known to be repressed by the mgrA transcription factor ${ }^{46,49,50}$, and two lines of evidence from our transcriptome experiment suggest that $m g r A$ plays a role in evolvability. First, $m g r A$ was overexpressed in low evolvability strains (two-sided Wald test: $\log 2$ fold-change $=0.885$, standard error $=0.206, t=-4.305$, adjusted $p=0.005597$, Supplementary data 3$)$. Second, we found a negative correlation between $m g r A$ and norA transcript levels across 12 of the 14 pairs of high and low evolvability strains (Supplementary Fig. 12, exact binomial test $P=0.00091, N=14$ ), suggesting that $m g r A$ represses norA in low evolvability strains. Although this relationship between norA and $m g r A$ is clear at a qualitative level, the quantitative correlation between $m g r A$ and nor $A$ transcript levels is weak (Supplementary Fig. 12), suggesting that other regulatory genes and/or post-translational modification of MgrA play important roles in mediating norA expression ${ }^{49-51}$. Interestingly, $m g r A$ is highly conserved, and we only identified two polymorphic sites in the strains used in our study. These polymorphisms showed no evidence of association with 
a

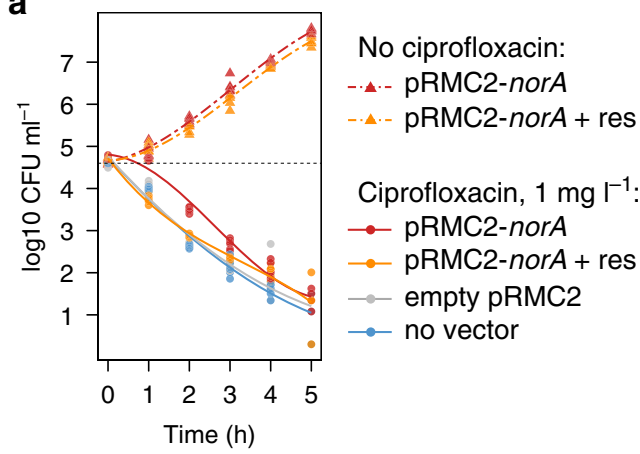

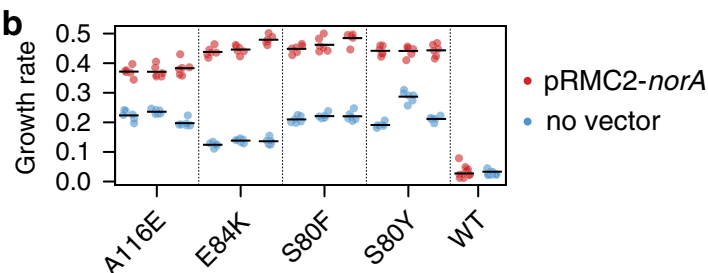

C

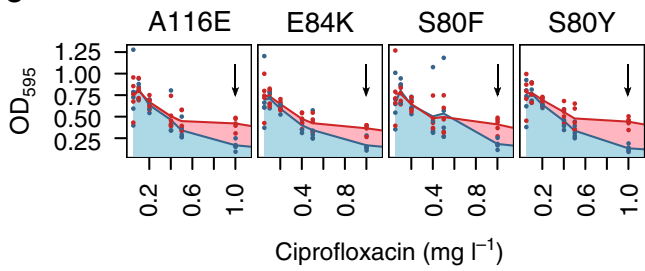

Fig. 5 The mechanism of norA potentiation. a Exponentially growing cells were exposed to $1 \mathrm{mg} \mathrm{I}^{-1}$ of ciprofloxacin and viable cells counts were estimated by plating on TSB agar. Four treatments were compared: (i) norA overexpression $=$ red solid line, (ii) norA overexpression and reserpine $(33 \mu \mathrm{M})$ inhibition $=$ orange solid line, (iii) empty vector control = grey line, and (iv) no vector control = blue line. In addition, cell density was measured in treatments (i) and (ii) without ciprofloxacin (red dash-dotted line and orange dash-dotted line, correspondingly). For each time-point, six independent replicates per treatment were measured (with the exception of the pRCM-norA cells in ciprofloxacin at $\mathrm{O} h$ which had $N=5$ ). The lines show model fit for polynomial regression $\left(F_{23,191}=622.6, p\right.$-value $\left.<2.2 \mathrm{e}-16\right)$. Post-hoc comparisons are shown in Supplementary Table 14. $\mathbf{b}$ Growth rate of grlA mutants with or without norA overexpression. We estimated the growth rate of the RN4220 wild type (WT) and three independently obtained mutants carrying each grlA substitution in the presence of $\left.1 \mathrm{mg} \mathrm{I}\right|^{-1}$ of ciprofloxacin. Growth rate in cells carrying pRMC2-norA (red) or cells without the vector (blue) was determined using growth curves shown in Supplementary Fig. 10b. Black horizontal lines show the mean growth rate of independent cultures ( $N=6$ per mutant/ treatment combination for A116E, E84K, S80F and S80Y, $N=11$ for WT pRMC2-norA and $N=12$ for WT no vector). Paired two-sided $t$-test comparing the means in the mutants with and without pRMC2-norA: $t=11.958$, d.f. $=11, p=1.206 \mathrm{e}-07$. Two-sided $t$-test comparing pRMC2-norA and no vector control in WT: $t=0.96281$, d.f. $=12.136, p=0.3544$. c Representative dose-response experiments for grlA mutants. Red lines show mean optical density with norA overexpression, and blue lines show mean density without overexpression. Black arrow shows the concentration used during experimental evolution. Five independent cultures were used per mutant/treatment/concentration. The results for all mutants are shown in Supplementary Fig. 10a. The difference of means between pRMC2-nor $A$ and no vector cells at $1 \mathrm{mg} \mathrm{I}^{-1}$ for all mutants was tested using two-sided paired $t-$ test: $t=-18.712, P=1.09 \mathrm{e}-09$, d.f. $=11$.

evolvability in our GWAS ( $P$ values $>10^{5}$ above conservative threshold), potentially providing evidence that the connection between $m g r A$ and evolvability is complex.

\section{Discussion}

We found that the rate and mechanisms of evolution of ciprofloxacin resistance vary dramatically across the diversity of $S$. aureus, an important human pathogen (Figs. 1,2). Studying the evolution of resistance at this broad scale using a combination of genomic and transcriptomic approaches allowed us to identify lineages with high evolvability and genes that potentiate the evolution of resistance, implying that it is possible to predict the evolution of resistance from genomic data. Remarkably, a single efflux pump gene (norA) plays a key role in accelerating the evolution of ciprofloxacin resistance, either by increasing resistance directly, as in the high evolvability CC398 lineage, or by increasing the benefit provided by classical ciprofloxacin resistance mutations that alter DNA topoisomerase.

NorA is a narrow-spectrum efflux pump that is known to contribute to the intrinsic ciprofloxacin resistance in S. aureus, but has not previously been linked to clinically relevant levels of resistance ${ }^{32,41,52,53}$. High evolvability strains of $S$. aureus show elevated levels of norA expression (Fig. 3), and overexpressing norA in a lab strain dramatically increases evolvability (Fig. 4b) by increasing the protective effect of 'classical' ciprofloxacin resistance mutations in grlA (Fig. 5b, c). Inhibiting NorA in clinical isolates leads to a marginal loss of intrinsic resistance (Fig. 4c) and a massive reduction in evolvability (Fig. 4d), suggesting that variation in norA expression creates a strong association between intrinsic resistance and evolvability across the diversity of S. aureus (Fig. 1).
Interestingly, positive epistasis has been found between ciprofloxacin resistance SNPs and mutations that increase drug efflux in Gram-negative bacteria ${ }^{54-57}$, suggesting that our findings may help to explain the evolution of ciprofloxacin resistance across a broad spectrum of bacterial pathogens where ciprofloxacin resistance has emerged as an important clinical problem, such as E. coli and $P$. aeruginosa. More generally, our findings suggest that more attention should be given to understanding interactions between resistance mutations and 'background' genetic variation that can epistatically modify the fitness effects of canonical resistance mutations ${ }^{14,30}$.

Although we have succeeded in linking norA expression to evolvability, we were not able to determine the underlying genetic basis of high evolvability outside of CC398. GWAS analysis has promising potential to uncover the genetic basis of bacterial traits, such as high resistance ${ }^{58}$ or virulence ${ }^{24}$, but this approach was unable to uncover any associations between SNPs and high evolvability. On the one hand, this lack of significance could be explained by the fact that high evolvability is driven by SNPs that are unique to individual lineages of $S$. aureus, in much the same way as lineage-specific IS elements potentiate the rapid evolution of resistance in CC398. Alternatively, it is possible that our analysis simply lacked the statistical power needed to uncover SNPs that are associated with high evolvability, perhaps as a result of limited recombination in $S$. aureus ${ }^{59}$. The negative correlation between the expression of the $m g r A$ repressor and evolvability suggests that $m g r A$-mediated repression of norA expression helps to constrain evolvability, and an important goal for future work will be to understand the connection between genetic variation, transcriptional regulation and evolvability in pathogenic bacteria.

Our study included a number of strains from clonal complex CC398, containing three sequence types-ST398 $(n=4)$, ST291 
$(n=7)$ and ST3535 $(n=1)$. The ST398 strains belong to the human-associated lineage of ST39860 and they evolved via mutations in grlA. The ST291 strains are genetically quite different from ST398 and represent a separate lineage within $\mathrm{CC} 38^{61}$; they are usually associated with humans ${ }^{62}$, but more recently isolated from livestock ${ }^{63,64}$. These ST291 strains (and the related ST3535 strain) rapidly and repeatedly evolved in response to ciprofloxacin by ISSaul-mediated tandem amplification of nor $A^{34}$, allowing them to increase ciprofloxacin resistance without any detectable fitness cost. This is perhaps surprising, given that the largest amplifications increased genome size by about 200 $\mathrm{kb}$, which is equivalent to $\mathrm{a} \sim 7 \%$ increase in genome size. However, a number of other studies have found evidence that $S$. aureus can acquire novel DNA, such as plasmids and SCCmec elements, without any additional detectable fitness burden ${ }^{65,66}$. More generally, the high copy number of ISSaul in CC398 suggests that gene amplification may be an important mechanism of adaptation to novel environments, such as new host species and antimicrobials ${ }^{67}$.

Whole-genome sequencing of bacterial pathogens is fundamentally transforming clinical microbiology 68,69 , and a key challenge for this field is to exploit the wealth of data provided by genomic sequences to better understand bacterial pathogenesis and epidemiology $3,23,24,70$. By uncovering the link between genotype and evolvability, it should be possible to use pathogen genomic data to help predict the likelihood that resistance will evolve during antibiotic treatment and to alter treatment strategies accordingly. For example, our work suggests that ciprofloxacin should be used cautiously to treat infections caused by CC398 (and in particular ST291), due to the high risk of de novo evolution of resistance. Outside of CC398, measuring the intrinsic ciprofloxacin resistance of clinical isolates could be used in conjunction with phylogenetic data obtained from genome sequencing (Fig. 1) to predict the risk of resistance evolution during treatment. For example, several clusters of strains in Fig. 1c have low evolvability given their intrinsic resistance, making these strains good candidates for ciprofloxacin treatment. Although we have focused on ciprofloxacin resistance in S. aureus, it should be possible to use experimental evolution and genomics to predict the risk of resistance evolution for other pathogen/drug combinations. This approach should be particularly useful to predict the evolution of resistance to novel antimicrobials prior to their introduction into clinical use $\mathrm{u}^{3,71}$.

Understanding the genetic basis of evolvability also opens up the possibility of using novel therapies to prevent the evolution of resistance. For example, we have shown that using an efflux pump inhibitor can prevent the evolution of resistance by blocking an important evolutionary path to low costs resistance (see also ref. ${ }^{14}$ ). Recent work has shown that efflux pumps can also accelerate resistance evolution by increasing the mutation rate $^{72}$ or facilitating the acquisition of plasmids ${ }^{73}$, suggesting that efflux pump inhibitors may have promising potential to suppress the evolution of resistance ${ }^{74}$ for a large number of combinations of pathogen and antibiotic.

\footnotetext{
Methods

Media and reagents. 96-well flat-bottom plates (Nunc, 260860) and MuellerHinton 2 medium (MH2 medium, Sigma-Aldrich, 90922) were used for culturing $S$. aureus, measuring antibiotic resistance and performing all evolution experiments. Tryptic Soy Broth (TSB medium; Sigma-Aldrich, 22092) was used for preparation of competent cells, for selecting transformed clones and for growing $S$. aureus prior to DNA isolation. Incubation was performed at $37^{\circ} \mathrm{C}$ and $225 \mathrm{rpm}$ orbital shaking inside a MaxQ 8000 shaking incubator (Thermo Fisher).

Ciprofloxacin (Sigma, 17850) was dissolved in water $\left(5 \mathrm{mg} \mathrm{ml}^{-1}\right)$ and stored at $-20^{\circ} \mathrm{C}$. The other reagents used in this study were chloramphenicol $\left(25 \mathrm{mg} \mathrm{ml}^{-1}\right.$ in ethanol, Acros Organics, 227920250), ampicillin (50 $\mathrm{mg} \mathrm{ml}^{-1}$ in water, Sigma, A1593), kanamycin sulfate $\left(25 \mathrm{mg} \mathrm{ml}^{-1}\right.$ in water, Fisher Chemicals), rifampicin
}

(50 $\mathrm{mg} \mathrm{ml}^{-1}$ in DMSO, Millipore, 557303), reserpine (10 $\mathrm{mg} \mathrm{ml}^{-1}$ in DMSO, Sigma-Aldrich, 83580).

S. aureus strains. In total, 222 strains of $S$. aureus were collected from colonized and infected patients in Oxford and Brighton, as previously described ${ }^{18}$. To ensure that these isolates were clonal, the strains were streaked out on TSB agar plates. A single colony was picked to inoculate overnight culture in TSB broth. The overnight cultures of 222 strains were mixed with glycerol to a final concentration of $15 \% \mathrm{v} / \mathrm{v}$ and stored at $-80^{\circ} \mathrm{C}$

Measuring intrinsic resistance by broth microdilution. Intrinsic resistance was determined for 222 strains by exposing five replicate cultures per strain to 8 doses of ciprofloxacin ( $>8800$ cultures in total). The strains were handled in five batches with up to 60 strains per batch. Five replicate plates were included for each batch/ dose combination, and each plate contained only one replicate of each strain. In order to minimize the effect of well location, five different strain layouts were created by randomization. As a result, every strain was exposed to a given ciprofloxacin concentration in five different plates and at five different well locations. To control for contamination, limit excessive evaporation and avoid an edge effect, 36 wells on the edge of each 96-well plate did not contain bacteria.

The strains were recovered from $-80^{\circ} \mathrm{C}$ stock by growing overnight cultures for $22 \mathrm{~h}$, diluted 1:100 and randomized across five 96-well plates using a Precision XS automatic pipetting station (BioTek). The resulting master plates were used for transferring $10 \mu \mathrm{l}$ of bacteria to assay plates containing $190 \mu \mathrm{l}$ of $\mathrm{MH} 2$ broth with ciprofloxacin. The assay plates were placed into the incubator for $22 \mathrm{~h}$. After incubation was completed, optical density was measured at $\mathrm{OD}_{595}$ using a Synergy 2 plate reader (BioTek).

$\mathrm{OD}_{595}$ values were used to estimate the MIC (minimal inhibitory concentration) and perform a dose-response analysis. A concentration was considered inhibitory if $3 / 5$ replicate cultures did not reach a cut-off value of 0.08 $\mathrm{OD}_{595}$ including a blank. The median blank value was $0.042 \mathrm{OD}_{595}($ s.d. $=0.003)$ however higher values were occasionally observed due to dust, excessive evaporation, etc. Two assay plates were excluded from the analysis because of unusually high variation in control wells (batch 3 , dose $=0.1$, replicate plate 5 , and batch 4 , dose $=0.025$, replicate plate 4 ). In the remaining dataset, only $2 / 6406$ control wells had $\mathrm{OD}_{595}>0.08$.

For the dose-response analysis, a non-linear 4-parameter model was fitted:

$$
f(x)=c+\frac{d-c}{1+\exp ^{[\mathrm{b} \times(\log (\mathrm{x})-\log (\mathrm{e}))]}},
$$

where $c$ is te lower asymptote (i.e. response variable at complete inhibition), $d$ is the upper asymptote (uninhibited growth), $e$ is an inflection point equivalent to a half growth inhibition dose $\left(\mathrm{IC}_{50}\right)$ and $b$ is a slope of the curve at the inflection point. Parameter $c$ was fixed at 0.08 to be consistent with the cut-off value, the remaining 3 parameters were estimated separately for each strain by fitting dose response curves using the drc package in $\mathrm{R}^{75}$. The standard errors for $\mathrm{IC}_{50}$ were obtained using the coeftest function from the package lmtest The dose response curves were also used to calculate the area under a curve (AUC) using the trapz function in the pracma package.

Experimental evolution of resistance. The evolvability of 222 ciprofloxacinsensitive strains (MIC $\leq 1 \mathrm{mgl}^{-1}$ ) was measured by exposing each strain to $1 \mathrm{mgl}^{-1}$ for 5 daily transfers. For each strain, 12 replicate populations were included, amounting to more than 2600 bacterial populations, which were transferred daily in 96-well plates. Prior to the experiment, replicates were distributed across the plates so that each plate had a maximum of one replicate of any strain (except for six plates which had two replicates for a set of 30 strains). For convenience, the strains were handled in sets of up to 60 strains. For each set of strains, three-randomized plate layouts were generated to assign strain locations within a plate. Each randomization layout was used for only four replicate plates. As a result, any given strain had replicate cultures located in 12 plates and in four different well locations (or in six plates for the set of 30 strains mentioned previously). Wells at the plate edges (rows $\mathrm{A}$ and $\mathrm{H}$, and columns 1 and 12) were used for blank measurements and as contamination controls.

The strains from $-80^{\circ} \mathrm{C}$ stock were grown overnight in $200 \mu \mathrm{l}$ of MH2 (a single culture per strain, five 96 -well plates in total). After $22 \mathrm{~h}$ of incubation, each plate was used to inoculate three fresh plates by transferring $20 \mu \mathrm{l}$ of bacteria to $180 \mu \mathrm{l}$ of MH2. This step was simultaneously used to randomize the locations of strains within plates and was performed using a BioTek Precision XS automatic pipetting station. The resulting 15 plates contained three replicate cultures per strain and had unique strain layouts. After $20 \mathrm{~h}$ at $37^{\circ} \mathrm{C}$, the cultures were transferred again to fresh plates with $\mathrm{MH} 2$ and returned to the incubator. After the third recovery transfer, $10 \mu \mathrm{l}$ of culture was inoculated into MH2 medium containing $190 \mu \mathrm{l}$ of $1 \mathrm{mgl}^{-1}$ of ciprofloxacin. Each culture was used to establish four replicate populations resulting in 12 populations per strain. The plates were incubated for $22 \mathrm{~h}$ until the next transfer. In total, five transfers were performed in $\mathrm{MH} 2$ medium with ciprofloxacin. At the end of each transfer, the optical density at $\mathrm{OD}_{595}$ was measured. By transfer 5, most populations had either gone extinct or evolved resistance and had a high density (more than $90 \%$ of surviving populations had an 
$\left.\mathrm{OD}_{595}>0.5\right)$. At this point, one more transfer without ciprofloxacin was performed and the evolved populations were frozen in $-80{ }^{\circ} \mathrm{C}$ in $15 \%$ glycerol.

Populations were considered to have survived if they had an $\mathrm{OD}_{595}>0.08$ (including blank) by transfer 5. Only $2 / 1942$ control populations had an optical density above 0.08 , indicating a detectable but very low error rate due to experimental artefacts and contamination. In the final dataset, evolvability was defined as the proportion of survived populations per strain, ranging from $0 / 12$ to $12 / 12$.

To determine whether intrinsic resistance is a good predictor for evolvability, a generalized linear model was fitted with a binomial family of distribution of errors. Numbers of survived and extinct populations per strain were used as a response variable (via the logit link function) and $\mathrm{IC}_{50}$ as a continuous covariate. In order to control for a lineage effect on evolvability, each strain was assigned to one of 14 phylogenetic clusters. In particular, the cluster package in R was used to perform PAM-clustering algorithm ("partition around medoids", https://cran.r-project.org/ package $=$ cluster). The performance of PAM-clustering was checked using the silhouette diagnostic plots provided by the same package. Similar clustering approaches have been used in previous studies to control for a lineage effect ${ }^{23,24}$. Phylogenetic clustering was performed using a genetic distance matrix calculated from a whole genome tree generated from sequence data mapped to MRSA252. Cluster identities and their interaction with the $\mathrm{IC}_{50}$-covariate were added to the model as fixed predictors. The emmeans package in $\mathrm{R}$ was used to test whether cluster-specific effects differed significantly from the effects calculated for the overall sample.

Ciprofloxacin resistance of evolved populations was determined by broth microdilution method. Evolved populations were selected to maximize phylogenetic diversity and variation in evolvability. Each population was derived from a different parental strain (except strain ERR418532 for which two populations were included). The growth of five replicate cultures for 83 evolved populations was assayed across eight different concentrations of ciprofloxacin, ranging from 0 to $32 \mathrm{mgl}^{-1}$. The assays were performed in two blocks using fiverandomized plate layouts. MIC, $\mathrm{IC}_{50}$, and $\mathrm{AUC}$ was calculated using $\mathrm{OD}_{595}$ after $22 \mathrm{~h}$ of incubation.

DNA extraction and sequencing. DNA was extracted from one representative evolved population for a subset of strains. In this subset, almost all the strains which had an evolvability $\leq 3 / 12(n=48)$ or $\geq 9 / 12(n=44)$ were included. Eight strains were excluded because they either failed to grow or the DNA concentration was too low for sequencing. In addition, one evolved population for a representative subset of strains with evolvability ranging from $4 / 12$ to $8 / 12(n=29)$ was included, resulting in a total selection of 121 evolved strains.

Strains were re-grown from $-80^{\circ} \mathrm{C}$ stock in $2 \mathrm{ml}$ of TSB. Cells were collected by centrifuging for $5 \mathrm{~min}$ at $5000 \mathrm{rpm}$ and re-suspended in enzymatic digest buffer (20 mM Tris-HCl, $2 \mathrm{mM}$ EDTA, $1.2 \%$ Triton-X100, $0.03 \mathrm{mg} \mathrm{ml}^{-1}$ lysostaphin (Sigma-Aldrich, L7386), $25 \mathrm{mg} \mathrm{ml}^{-1}$ chicken egg lysozyme (Sigma-Aldrich, 62970)). After $2-4 \mathrm{~h}$ of incubation at $37^{\circ} \mathrm{C}$ to digest the cell wall, DNA was extracted using DNeasy Blood \& Tissue kit (Qiagen) following the manufacturer's protocol for Gram-positive bacteria. The integrity of genomic DNA was checked on $0.9 \%$ agarose gel and the concentration was measured using a QuantiFluor dsDNA Kit (Promega).

All parental strains were previously sequenced ${ }^{76}$ using an Illumina HiSeq 2000 platform (San Diego, CA, USA) and downloaded from the European Nucleotide Archive Sequence Read Archive (study accession number: PRJEB5261). Evolved strains were sequenced using an Illumina HiSeq 4000 platform with 150 base pair (bp) paired-end reads at the Oxford Genomics Centre (Wellcome Centre for Human Genetics, University of Oxford, Oxford, UK). Sequencing of evolved strains yielded a mean coverage of $302 x$.

Sequencing reads from both the parental and evolved strains were trimmed using Trimmomatic v0.3677. Leading and trailing bases were trimmed if the Phred quality score was less than 20 and a 4-base wide sliding window was used to cut reads when the average quality per base dropped below 15 . Any read less than $50 \mathrm{bp}$ long was dropped. Sequences were mapped to the ST36 reference strain MRSA252 (GenBank accession no. BX571856.1) using Stampy v1.0.3178, with an expected substitution rate of 0.01. Single nucleotide polymorphisms (SNPs) were called using the SAMtools v1. $7^{79}$ mpileup command, with the following command line options: -F 0.002 -g -t DP -t SP, and the bcftools v1.780 call command with the following command line options: - $\mathrm{m}-\mathrm{O} v-\mathrm{M}-\mathrm{P} 0.001-\mathrm{p}$ 0.5. All SNPs supported by fewer than 5 reads or by more than twice the mean sequencing depth (calculated across all sites in the genome) were filtered from the consensus FASTA sequence, which was generated using the bcftools consensus command. Non-unique regions in the reference genome were identified by self-self BLAST analysis of the reference with a word size of 28 , using the megablast algorithm in BLAST v2.5.0 ${ }^{81}$. Such regions represented $5.93 \%$ of the genome and were filtered from the consensus FASTA sequence for each strain. Any base with a heterozygous call under a diploid model was also filtered. Sequences were assembled into de novo contigs for each genome using SPAdes v3.11.1 with the -careful mode turned on ${ }^{82}$. Multi-locus sequence typing was performed on the assemblies using the S. aureus MLST website (https:// pubmlst.org) sited at the University of Oxford ${ }^{83}$.
Phylogenetic analysis of full parental sequence dataset. After mapping to the MRSA252 reference genome, genome sequence data were used to construct a maximum likelihood phylogenetic tree in RAxML v8.2.9 ${ }^{84}$, using the GTRCAT model of nucleotide substitution. Branch lengths were corrected for recombination using ClonalFrameML ${ }^{85}$, with a transition/transversion rate ratio (kappa) value of 4.04. This value of kappa was estimated from four independent analyses of subsets of the data $(n=50)$ in PhyML v 3.3.20170105

Determination of evolved SNPs. Sequence assemblies for the parental strains were annotated using Prokka 1.12-beta ${ }^{87}$. Snippy v4.0-dev2 (https://github.com/ tseemann/snippy) and breseq v0.33.188 were used to identify SNPs between each annotated parental genome and the sequence reads from its corresponding evolved genome for 121 strains. Snippy uses bwa to align reads to a reference (here, the assembled parental genome) and FreeBayes to call variants ${ }^{89,90}$. SNPs were required to have a minimum read mapping quality of 60 , minimum base quality of 30 and a minimum coverage of 30 . A lower mapping coverage threshold (15) was used for SNPs in ancestral isolates due to the lower depth at which these were sequenced. Snippy does not call SNPs at sites with heterozygous genotypes, which here represent diversity within the sequenced culture. This means that any minor variants present in the parental population that subsequently rose to fixation in the evolved population are reported as evolved SNPs by Snippy. As such, only those SNPs reported by both Snippy and breseq, and located at sites without a heterozygous call in the parental strain, were classified as evolved SNPs. In 15/121 strains, no SNPs were identified in genes for established ciprofloxacin resistance targets (topoisomerase IV, DNA gyrase, norA). 6/15 strains were ST291/ST3535, for which resistance is inferred to be conferred by amplification of norA. Inspection of the variant call format (VCF) file revealed that four of the remaining nine strains had heterozygous calls at known ciprofloxacin resistance sites, suggesting that these evolved populations represented a mix of resistant and susceptible clones. These strains were, therefore, classified as resistant. No known resistance mechanism could be identified in five strains.

Copy number estimation of amplified region. Sequence data were remapped to the ST291 reference genome JP80 (GenBank accession no. AP017922.1) to obtain sequence depth estimates for the flanking transposase genes (not present in MRSA252). Copy number was estimated as the total number of good quality reads mapping at each site, normalized by the mean depth across all sites in the mapped genome.

Evolutionary analysis of norA. To explore genetic diversity of norA within the dataset, BLAST was used to identify the norA gene and $250 \mathrm{bp}$ region upstream of norA in the sequence assembly for each parental strain. Sequences were aligned using Muscle v3.8.31 and a maximum likelihood phylogenetic tree was constructed in PhyML using an HKY85 model of nucleotide substitution. Tree topology improvement was computed using nearest-neighbour interchange and subtree pruning and regrafting, selecting the best from both searches. This analysis identified three genetically distinct clades within the phylogeny, from which cladespecific consensus sequences were generated by taking the majority allele at each site. All SNPs within the promoter region for each sequence relative to the respective clade-specific consensus and occurring at the tips of the whole genome phylogeny were tested for association with norA expression, where this was measured. Ten SNPs were identified in total, five of which were in strains with expression data across all three clades in the norA phylogenetic tree.

Copy number estimation of ISSau1 in parental strains. Sequence data from all parental strains were mapped to the JP02758_0628 transposase gene located at the $5^{\prime}$ end of the amplified region of the genome using Stampy. Depth of sequence coverage at each site was calculated using the samtools depth program. Transposase copy number was estimated by normalizing the mean sequence depth across all mapped sites by the mean sequence depth across all sites in the corresponding genome mapped to MRSA252.

Mapping the genomic location of new copies of transposase gene. The increase in transposase copy number can be a result of tandem amplification or transposition to a different genomic location. To differentiate these two mechanisms, the location of the JP02758_0628 transposase gene was mapped in eight parental and eight evolved strains, in which the transposase amplification was detected. This analysis was carried out using ISmapper v2.0.135. ISMapper takes unmapped paired reads, maps them to an IS element and, then, identifies pairs in which only one read is mapped to the IS element (JP02758_0628). These partially mapped pairs are assumed to be at the junction of a transposon and its genomic neighbourhood, and later used to determine transposition sites within a reference genome (JP80, GenBank accession no. AP017922.1). As a result, a consistent pattern of JP02758_0628 locations was detected between ancestral and evolved sequences, identifying no new transposition sites in the evolved strains and supporting the hypothesis of tandem amplification. 
Genome-wide association study. A GWAS was carried out using the bugwas ${ }^{70}$ package in $\mathrm{R}^{91}$. bugwas uses a linear mixed model to test for phenotype associations with both SNPs and lineages, while controlling for relatedness between samples. As input, bugwas requires a sequence alignment, phylogenetic tree and phenotype data for each sample. While it is possible to perform a continuous trait GWAS in bugwas, the evolvability phenotype was not normally distributed and therefore a continuous trait model was not suitable for this dataset. As such, a binary trait GWAS was performed by limiting the analysis to low (evolvability $\leq 2$ / 12 ) and high (evolvability $\geq 9 / 12$ ) evolvability strains, encoding phenotypes as either 0 or 1 respectively. All strains with intermediate evolvability estimates were excluded from the GWAS analysis, leaving a dataset of 96 strains. A sequence alignment for all strains was obtained by mapping the corresponding sequence data to the MRSA252 reference genome (as described above). A phylogenetic tree was constructed from this sequencing alignment in RAxML v8.2.9 ${ }^{84}$ using the GTRCAT model of nucleotide substitution. 89,874 biallelic and 8,591 multiallelic SNPs were tested for association with evolvability and annotated in bugwas using the MRSA252 reference genome. $P$-values were calculated using a likelihood ratio test and a Bonferroni correction was applied.

Transcriptome analysis of parental strains. RNA-Seq analysis was performed using the RNA from 30 parental strains. Since differences in gene expression may be confounded by phylogenetic relationship, especially between phylogenetically distant strains, expression was measured for 15 pairs of closely related high and low evolvability strains. First, 15 high evolvability parental strains (evolvability $\geq 9 / 12$ ) were chosen to maximize phylogenetic diversity. For each high evolvability strain, the most closely related low evolvability strain (evolvability $\leq 1 / 12$ ) was identified (Supplementary Table 6). The strains were grown for $16 \mathrm{~h}$ overnight in $\mathrm{MH} 2$ broth at $37^{\circ} \mathrm{C}$ at $225 \mathrm{rpm}$, diluted 1:20 in a fresh pre-warmed $\mathrm{MH} 2$ and incubated for an additional $1 \mathrm{~h}$ and $20 \mathrm{~min}$ until they reached a density of $0.160-0.170 \mathrm{OD}_{595}$. Ciprofloxacin was added to achieve a final concentration of $1 \mathrm{mgl}^{-1}$. After $1.5 \mathrm{~h}$, the exposure to ciprofloxacin was stopped by spinning cells for $10 \mathrm{~min}$ at $7000 \mathrm{rpm}$, decanting supernatant and re-suspending the cells in $200 \mu \mathrm{l}$ of PBS. Finally, $500 \mu \mathrm{l}$ of RNA-protect Bacteria Reagent (Qiagen) was added and the cells were equilibrated at room temperature for $5 \mathrm{~min}$ and then frozen at $-80^{\circ} \mathrm{C}$. The cells in RNA-protect solution were thawed, centrifuged for $10 \mathrm{~min}$ at $5000 \mathrm{rpm}$ and separated from the supernatant. Next, the cells were mixed with $100 \mu \mathrm{l}$ of enzymatic digest buffer $\left(30 \mathrm{mM}\right.$ Tris- $\mathrm{HCl}, 1 \mathrm{mM}$ EDTA, $2 \mathrm{mg}^{-1}$ protease $\mathrm{K}$ (Thermo Fisher), $3.25 \mathrm{mg} \mathrm{l}^{-1}$ lysozyme (Sigma-Aldrich, 62970) and $0.15 \mathrm{mg} \mathrm{l}^{-1}$ lysostaphin (Sigma-Aldrich, L7386)), re-suspended by pipetting and incubated for $10 \mathrm{~min}$ at room temperature with shaking. Three hundred and fifty microliters of RTL buffer (containing 2-mercaptoethanol (Sigma-Aldrich, M3148)) was added and RNA extraction was performed using RNeasy Mini Kit (Qiagen), following the manufacturer's protocol and including the optional step of DNase I on-column digestion. Elution was performed twice using the same volume of $50 \mu \mathrm{l}$ of RNase-free water. RNA concentration was determined using the QuantiFluor RNA System (Promega). Samples were sent to Oxford Genomics Centre (Wellcome Centre for Human Genetics, University of Oxford, Oxford, UK), where rRNA depletion was performed prior to sequencing using an Illumina HiSeq 4000 platform, generating 75 bp paired-end reads.

Initial principal component analysis identified a likely batch effect, affecting one high evolvability strain. This strain and its low evolvability pair was removed from subsequent analysis. Transcript quantification of the RNA-Seq data from the remaining 14 pairs was performed using Salmon v0.11.3, which maps reads to an indexed reference transcriptome (MRSA252; GenBank accession no. BX571856.1) using quasi-mapping approach and an inbuilt correction for fragment-level GC bias $^{92}$. Indexing of the reference transcriptome removed sequence-identical duplicate transcripts and used a kmer length of $31 \mathrm{bp}$. The DESeq2 library in R was used to estimate gene counts and perform differential expression analysis ${ }^{93}$ in Bioconductor, with the requirement of at least 1 read mapping to each gene for every strain. Normalised norA transcript counts for individual strains were obtained using the plotCounts function in the DESeq2 library in R.

To test whether genes in different KEGG pathways were up or downregulated in high or low evolvability strains, a KEGG pathway enrichment analysis was carried out using the kegga and topKEGG functions in the limma library in R. A false discovery rate cutoff for differentially expressed genes of 0.05 was used. A given KEGG pathway was considered to be significantly enriched when $P<0.05$.

Mutation rate estimation (fluctuation test). Mutation rate was determined for 33 parental strains. These 33 strains were selected in the following way. First, 11 high evolvability strains (survival $>10 / 12$ ) were chosen to maximize phylogenetic representation. Second, each of these strains was paired to the closest low evolvability strain (survival $\leq 1 / 12$ ). In addition, 11 strains representing CC398 (ST398, ST291 and ST3535) were included. The strains were assigned to three experimental blocks, ensuring a similar representation of high and low evolvability strains in each block.

The strains were re-grown from $-80^{\circ} \mathrm{C}$ stock in $200 \mu \mathrm{l}$ of $\mathrm{MH} 2$ medium. After $24 \mathrm{~h}$ of incubation, strains were subject to a bottleneck by diluting them $10^{7}$ times. The bottleneck step was required to remove any pre-existing low frequency mutations. The diluted bacterial cultures were used to establish 16 replicate cultures per strain in $1.2 \mathrm{ml}$ deep well plates (Brand, 701340) with $300 \mu$ l of MH2 per well.
After overnight incubation at $37^{\circ} \mathrm{C}, 5 \mu \mathrm{l}$ were sampled from eight replicates, diluted $10^{7}$ or $10^{8}$ times and plated on TSB agar plates to estimate population density. Two hundred microliters of overnight cultures from all 16 replicates per strains were plated on TSB-agar plates containing $100 \mathrm{mgl}^{-1}$ of the antibiotic rifampicin. After $24 \mathrm{~h}$, the TSB plates without antibiotic were photographed using a ColonyDoc-It Imaging Station (UVP, Cambridge, UK). The plates with antibiotic and containing rifampicin-resistant mutants were photographed after $48 \mathrm{~h}$. Colonies were counted in Image ${ }^{94}$ using a custom script written in ImageJ macro language.

The colony counts from both types of plates were imported into R. The mutation rate was calculated using the rSalvador package in $\mathrm{R}$, which provides a maximum likelihood estimate of mutation rate under the Luria-Delbruck model and accounts for variation in population density ${ }^{95}$. To compare mutation rates between strains, a Wilcoxon signed rank test (paired, two-sided) was performed.

The effect of reserpine on ciprofloxacin resistance. To determine the effect of the nor A inhibitor reserpine on intrinsic resistance and evolvability, 27 parental strains (out of 222) were chosen using the following algorithm. First, nine high evolvability strains (survival $>10 / 12$ ) were selected in different parts of the phylogenetic tree. Then, for each high evolvability strain the closest low evolvability strains was identified (survival $\leq 1 / 12$ ). In addition, we included nine strains from the high evolvability clonal complex CC 398 . This set of strains is a subset from the 33 strains used for measuring mutation rates. However, this set of 27 strains is different from the set used in transcriptomic analysis (though, two sets are overlapping), because of different survival thresholds used for defining high and low evolvability strains and because here we included CC398 strains.

The strains were recovered from $-80^{\circ} \mathrm{C}$ stock and re-grown for two transfers in $200 \mu \mathrm{l}$ of MH2 broth. During the second transfers, strain layouts were randomized on plates using a Precision XS automated pipetting station (BioTek). Five different randomization layouts were used corresponding to five replicate cultures included per dose/treatment combination. Half of the cultures were exposed to eight different concentrations of ciprofloxacin, and half to the same antibiotic concentration and $33 \mu \mathrm{M}$ of reserpine. After $22 \mathrm{~h}$ of incubation, population growth was measured using a plate reader $\left(\mathrm{OD}_{595}\right)$.

A dose-response analysis was performed by fitting a 4-parameter model. The dose-responses curves were used to estimate $\mathrm{IC}_{50}$. The effect of reserpine on $\mathrm{IC}_{50}$ was evaluated by using the function EDcomp from the R package drc (version 3.01). In addition, $\mathrm{IC}_{50}$ was compared using a two-sided Wilcoxon signed-rank test.

The effect of reserpine on evolvability. To estimate the effect of efflux pump inhibitor on evolvability, experimental evolution was performed by exposing $S$. aureus strains to $1 \mathrm{mg}^{-1}$ of ciprofloxacin, either with or without $33 \mu \mathrm{M}$ of reserpine. The same 27 strains that were used for measuring the effect of reserpine on intrinsic resistance were used here. For each strain, 16 replicates with reserpine and 16 replicates without reserpine were included. Strain positions on plates were randomized using four different plate layouts. The wells at the plate edges (i.e. rows $\mathrm{A}$ and $\mathrm{H}$ and columns 1 and 12) were used as controls.

The strains from $-80^{\circ} \mathrm{C}$ stock were re-grown overnight. During the second transfers, strain positions within the plate were randomized using an automated pipetting station. Half of the populations were challenged with $1 \mathrm{mgl}^{-1}$ of ciprofloxacin, and the other half with $1 \mathrm{mgl}^{-1}$ ciprofloxacin and $33 \mu \mathrm{M}$ reserpine. Every day, $>800$ populations were transferred to fresh medium containing the same dose of ciprofloxacin and/or reserpine. Population growth was measured after each transfer by reading optical densities $\left(\mathrm{OD}_{595}\right)$ using a plate reader. The experiment was completed after five transfers.

Due to incomplete solubility of reserpine in water, the optical density of $\mathrm{MH} 2$ with reserpine was slightly higher than without reserpine. The average increase in optical density due to reserpine was $0.011 \mathrm{OD}_{595}$, as estimated by comparing a blank sample with and without reserpine $(0.049 \pm 0.002$ and $0.060 \pm 0.008$, correspondingly; median \pm s.d.). Therefore, prior to analysis, measurements from wells containing reserpine were corrected by subtracting 0.011 . After applying the correction, populations that reached a density greater than $0.08 \mathrm{OD}_{595}$ were considered to have survived. The difference in survival due to the presence/absence of reserpine was analysed separately for each strain by performing a Fisher's exact test. The resulting $p$-values were adjusted using Holm-Bonferroni method $(n=27)$.

Cloning norA into the pRMC2 expression vector and transformation. Vector pRMC2 was obtained from Addgene (\#68940) ${ }^{96}$. The pRMC2 was linearized with EcoRI and KpnI restriction enzymes (NEB) and gel purified using the QIAquick Gel Extraction Kit (Qiagen). The norA gene and a 69 bp upstream region was amplified with PCR, using Phusion DNA Polymerase (NEB). The genomic DNA of strain ERR418607 (ST398) was used as a template. The primers contained 5' overhangs and were designed for Gibson assembly (see Supplementary Table 15) The linearized vector and the PCR product were assembled using NEBuilder HiFi DNA Assembly Kit (NEB) and transformed into E. coli DC10B (BCCM, Belgium, LMBP 9585). The clones were selected on LB plates with ampicillin $\left(100 \mathrm{mgl}^{-1}\right)$, screened using PCR and confirmed by Sanger sequencing (primers are listed in Supplementary Table 15). The expression vector containing norA was transformed 
into S. aureus RN4220 (DSMZ, Germany, DSM 26309) by electroporation using the protocol described in ref. ${ }^{40}$. The S. aureus transformants were selected on TSB agar plates with chloramphenicol $\left(10 \mathrm{mgl}^{-1}\right)$ and verified by PCR and Sanger sequencing.

\section{norA overexpression experiments (resistance and evolvability to cipro-} floxacin). The effect of norA overexpression on ciprofloxacin resistance was measured using the broth microdilution method. Although norA was cloned under a tetracycline-inducible promoter, increased resistance was also observed without adding the inducer. This is potentially because a native promoter was also cloned as a part of the norA upstream region. Further induction of norA with an anhydrotetracycline inducer increased resistance but simultaneously affected RN4220 growth rate in the absence of the antibiotic. In order to avoid a negative effect of high overexpression on bacterial fitness and the antimicrobial effect of anhydrotetracycline itself, we decided not use the inducer in all following experiments and relied on the uninduced level of expression.

To assess the effect of norA overexpression on resistance, the growth of RN4220 cells carrying pRMC2-norA was compared with cells carrying an empty vector and cells without vector. First, S. aureus strains were cultured overnight in MH2 (cultures that contained the plasmid were supplemented with $10 \mathrm{mgl}^{-1}$ chloramphenicol). The next day, cultures were adjusted to a density of $5 \times 10^{6} \mathrm{CFU} \mathrm{ml}^{-1}$ and inoculated into $\mathrm{MH} 2$ medium containing 10 different doses of ciprofloxacin. After $22 \mathrm{~h}$ of incubation, optical densities were measured. Three replicate cultures were included per dose/genotype combination. The effect of overexpressing norA was analysed using a dose-response curve.

To measure the effect of norA overexpression on evolvability, the RN4220 cells carrying pRMC2-norA or an empty vector pRMC2, and vector-free cells were experimentally evolved for five transfers at $1 \mathrm{mg} \mathrm{l}^{-1}$ of ciprofloxacin. Prior to the experiment, 40 colonies per genotype were selected to establish parallel cultures. After $22 \mathrm{~h}$ of incubation in $\mathrm{MH} 2$ broth (cultures with the plasmid were supplemented with chloramphenicol). Ten microliters of overnight culture were inoculated to wells containing $190 \mu \mathrm{l}$ of MH2 and $1 \mathrm{mg} \mathrm{l}^{-1}$ of ciprofloxacin. The cultures were transferred daily to a fresh medium with ciprofloxacin. Populations that reached an optical density of 0.08 or higher by transfer 5 were considered to be alive. To test the differences in evolvability between genotypes, Fisher's exact test was performed on the number of survived/extinct populations and $p$-values were corrected using the Holm-Bonferroni correction.

Killing assay using ciprofloxacin (the effect of norA expression on survival). To measure the effect of norA expression on cell survival during the exposure to ciprofloxacin, a killing assay was performed including four ciprofloxacin treatments $\left(1 \mathrm{mgl}^{-1}\right)$ and two control treatments (no ciprofloxacin). The ciprofloxacin treatments were: (i) the RN4220 cells overexpressing norA, (ii) the RN4220 cells overexpressing norA with $33 \mu \mathrm{M}$ reserpine (NorA inhibitor), (iii) the RN4220 carrying the empty vector pRMC2 and (iv) RN4220 cells with no vector. The control treatments (no ciprofloxacin) were (v) the RN4220 cells overexpressing norA and (vi) the RN4220 cells overexpressing norA supplemented with $33 \mu \mathrm{M}$ reserpine. A day before the experiment, overnight cultures were prepared in $\mathrm{MH} 2$ broth including $10 \mu \mathrm{g} \mathrm{ml}^{-1}$ of chloramphenicol for the bacteria carrying vector pRMC2. The overnight cultures were diluted 1:10 in pre-warmed $\mathrm{MH} 2$ broth and incubated for $2.5-3 \mathrm{~h}\left(225 \mathrm{rpm}, 37^{\circ} \mathrm{C}\right)$ until they entered an exponential growth phase. Next, all strains cells were diluted 1:5 times in pre-warmed $\mathrm{MH} 2$, the cell densities were brought to $\mathrm{OD}_{595}=0.072$ in $100 \mu \mathrm{l}$ of $\mathrm{MH} 2$ (including blank). The adjusted cultures were diluted 1:1000 using the pre-warmed media corresponding to their treatments (i.e. with or without $1 \mathrm{mgl}^{-1}$ ciprofloxacin and with or without $33 \mu \mathrm{M}$ reserpine) resulting in $\sim 5 \times 10^{4} \mathrm{CFU} \mathrm{ml}^{-1}$. The cell suspensions were transferred into pre-warmed 96 deep well plates (Brand, 701340) with $250 \mu \mathrm{l}$ per well, sealed with gas-permeable foil and incubated at $225 \mathrm{rpm}$ at $37^{\circ} \mathrm{C}$. Every hour, six replicate cultures per treatment were sampled, diluted in PBS buffer and plated to MH2 agar plates. For each time point, new cultures were sampled to avoid repeated measurements of the same cultures. The agar plates were incubated at $37^{\circ} \mathrm{C}$ for $24 \mathrm{~h}$ and, then, photographed using a ColonyDoc-It Imaging Station (UVP, Cambridge, UK). Colonies were counted with Image ${ }^{94}$ using a custom script written in ImageJ macro language.

\section{Obtaining spontaneous grlA mutants. Two hundred microliters of several} independent overnight cultures of the S. aureus RN4220 were plated onto MH2 agar plate containing either 1 or $2 \mathrm{mgl}^{-1}$ ciprofloxacin. Twenty-two colonies were isolated for DNA extraction and Sanger sequencing to identify resistance mutations. The primers used to amplify known fluoroquinolone resistance regions in grlA, gyrA, grlB and gyrB genes are listed in Supplementary Table 15. Twenty out of 22 mutants had grlA mutation (one A116P, seven A116E, three E84K, four S80Y and five S80F). For further characterization, three independently isolated mutants (i.e., isolated from different overnight cultures) were selected to represent four resistant mutations (A116E, E84K, S80Y and S80F) resulting in total 12 ciprofloxacin-resistant mutants of RN4220.

Transformation of grlA mutants with pRMC2-norA. Electro-competent cells were prepared for 12 grlA RN4220 following the protocol from ${ }^{40}$. The competent cells were transformed with the pRMC2-norA expression vector. The transformants were selected on TSB-plates supplemented with $10 \mu \mathrm{g} \mathrm{ml}^{-1}$ of chloramphenicol and confirmed by PCR and Sanger sequencing (primers are listed in Supplementary Table 15).

Measuring maximum growth rate of grlA mutants. To estimate the effect of norA overexpression on growth rate of the grlA mutants, 12 mutants carrying pRMC2-norA vector and the 12 corresponding parental mutants having no vector were compared. In addition, the wild-type RN4220 was included as a control (with or without pRMC2-norA). The mutants were grown overnight in MH2 broth (containing $10 \mu \mathrm{g} \mathrm{ml}^{-1}$ of chloramphenicol wherever necessary for pRMC2-norA selection). The overnight cultures were diluted 1:1000 in the MH2 broth with $1 \mathrm{mgl}^{-1}$ of ciprofloxacin and distributed in a 96-well plate with $200 \mu \mathrm{l}$ of cell suspensions per well. The assay plates were placed into a Synergy 2 plate reader (BioTek) and incubated at $37^{\circ} \mathrm{C}$ with continuous shaking. The bacterial growth was recorded by measuring the optical density $(\lambda=595 \mathrm{~nm}$ ) every $10 \mathrm{~min}$ for minimum $14 \mathrm{~h}$. For each mutant, six replicate cultures were included, and the experiment was performed in several blocks to accommodate all replicates.

Growth curves were used to estimate the maximum growth rate. A linear regression was used to calculate a slope for each 5 time-point interval of a growth curve in a sliding window fashion (by moving a window one time-point and repeating regression). The resulting distribution of slopes was used to find a maximum growth rate (corresponding to a maximum change in optical density per time unit (an hour)). Because the optical density data were log2-transformed prior the analysis, the obtained estimates should be equivalent to a number of cell divisions per hour (assuming linear relationship between the optical density and cell density during the log growth phase).

Measuring resistance to ciprofloxacin of grlA mutants. Twelve grlA mutants without vector and their corresponding transformants carrying the pRMC2-norA vector were cultured overnight in $\mathrm{MH} 2$ broth. Cultures that contained the vector were supplemented with $10 \mathrm{mgl}^{-1}$ chloramphenicol. The next day, cultures were adjusted to a cell density of $5 \times 10^{6} \mathrm{CFU} \mathrm{ml}^{-1}$ and inoculated into $\mathrm{MH} 2$ medium containing 13 different doses of ciprofloxacin $\left(0.01-12 \mathrm{mg} \mathrm{m}^{-1}\right)$. After $22 \mathrm{~h}$ of incubation, optical densities were measured using a plate reader. Five replicate cultures were included per dose/genotype combination. The effect of norA overexpression was analysed using a dose-response analysis and, additionally, by performing two-sided paired $t$-test for mean optical densities of mutants obtained at $1 \mathrm{mgl}^{-1}$ of ciprofloxacin.

Measuring maximum growth rate of strains with norA amplification. To estimate the effect of norA amplification on fitness, eight parental strains (before amplification) and eight evolved strains (after amplification) were assayed by determining maximum growth rate. These assays were performed in the absence of ciprofloxacin. The parental and evolved strains were grown overnight, diluted 1:1000 in the MH2 broth and inoculated in a 96-well plate $(200 \mu \mathrm{l}$ of cell suspensions per well). The assay plates were incubated inside of a Synergy 2 plate reader (BioTek) at $37^{\circ} \mathrm{C}$ with continuous shaking. The bacterial growth was recorded by measuring the optical density $(\lambda=595 \mathrm{~nm})$ every 10 min for minimum $14 \mathrm{~h}$. For each strain, three or four replicate cultures were included. Growth curves were used to estimate the maximum growth rate by calculate a slope for each 5 time-point interval of a growth curve in a sliding window fashion. The maximum slope is equivalent to the number of cell divisions per hour during the exponential growth phase in the absence of ciprofloxacin. Maximum growth rate in parental and evolved strains was compared using two-sided paired Wilcoxon sign rank test.

Statistics and reproducibility. Experimental evolution of 222 strains was repeated twice. The first attempt failed due to experimental error. Experimental evolution with cell expressing norA and experimental evolution with norA inhibitor were performed one time. The transcriptome experiment and fluctuation test were performed one time. The determination of resistance to ciprofloxacin in 222 parental strains, in 83 evolved populations, in 27 strains with reserpine, and in 12 grlA mutants were performed one time. The experiment with norA overexpression in RN4220, the killing assay and estimation of growth rates were performed one time after smaller trial experiments. The results from the trial experiments were reproducible.

Reporting summary. Further information on research design is available in the Nature Research Reporting Summary linked to this article.

\section{Data availability}

Whole-genome data for 222 parental strains are available from the European Nucleotide Archive, PRJEB5261. Reference genome sequence of S. aureus MRSA252 is available from the GeneBank, BX571856.1. Reference genome sequence of $S$. aureus JP080 is available from the GeneBank, AP017922.1. Whole-genome sequence data of 122 evolved strains were deposited at the Sequence Read Archive, BioProject PRJNA633882. RNA sequencing data for 28 strains are available from Gene Expression Omnibus database, GSE150762. 
Other data that support the findings of this study are available from figshare [https://doi. org/10.6084/m9.figshare.c.4984364]. Source data are provided with this paper.

\section{Code availability}

A Jupyter notebook showing the processing of next-generation sequencing data and a script used for counting bacterial colonies are available from Gitlab repository [https:// gitlab.com/apson/sa_evolvability/].

Received: 22 January 2020; Accepted: 14 July 2020;

Published online: 07 August 2020

\section{References}

1. O'Neill, J. I. M. Antimicrobial resistance: tackling a crisis for the health and wealth of nations. Rev. Antimicrob. Resist. 1, 1-16 (2014).

2. Bell, G. \& MacLean, C. The search for evolution-proof antibiotics. Trends Microbiol. 26, 471-483 (2018).

3. Sommer, M. O. A., Munck, C., Toft-Kehler, R. V. \& Andersson, D. I. Prediction of antibiotic resistance: time for a new preclinical paradigm? Nat. Rev. Microbiol. 15, 689-696 (2017).

4. Baym, M., Stone, L. K. \& Kishony, R. Multidrug evolutionary strategies to reverse antibiotic resistance. Science 351, aad3292 (2016).

5. Klemm, E. J., Wong, V. K. \& Dougan, G. Emergence of dominant multidrugresistant bacterial clades: lessons from history and whole-genome sequencing. Proc. Natl Acad. Sci. USA 115, 12872-12877 (2018).

6. Ben Zakour, N. L. et al. Sequential acquisition of virulence and fluoroquinolone resistance has shaped the evolution of Escherichia coli ST131. mBio 7, e00347-00316 (2016).

7. Dai, Y. et al. Decreasing methicillin-resistant Staphylococcus aureus (MRSA) infections is attributable to the disappearance of predominant MRSA ST239 clones, Shanghai, 2008-2017. Emerg. Microbes Infect. 8, 471-478 (2019).

8. Kitchel, B. et al. Molecular epidemiology of kpc-producing Klebsiella pneumoniae isolates in the United States: clonal expansion of multilocus sequence type 258. Antimicrob. Agents Chemother. 53, 3365-3370 (2009).

9. Logan, L. K. \& Weinstein, R. A. The epidemiology of carbapenem-resistant Enterobacteriaceae: the impact and evolution of a global menace. J. Infect. Dis. 215, S28-S36 (2017).

10. Pitout, J. D. D. \& DeVinney, R. Escherichia coli ST131: a multidrug-resistant clone primed for global domination. F1000Res. 6, 195 (2017).

11. Alhashash, F., Weston, V., Diggle, M. \& McNally, A. Multidrug-resistant Escherichia coli bacteremia. Emerg. Infect. Dis. 19, 1699-1701 (2013).

12. Baker, S., Thomson, N., Weill, F. X. \& Holt, K. E. Genomic insights into the emergence and spread of antimicrobial-resistant bacterial pathogens. Science 360, 733-738 (2018).

13. Ford, C. B. et al. Mycobacterium tuberculosis mutation rate estimates from different lineages predict substantial differences in the emergence of drugresistant tuberculosis. Nat. Genet. 45, 784-790 (2013).

14. Gifford, D. R. et al. Identifying and exploiting genes that potentiate the evolution of antibiotic resistance. Nat. Ecol. Evol. 2, 1033-1039 (2018).

15. Blount, Z. D., Barrick, J. E., Davidson, C. J. \& Lenski, R. E. Genomic analysis of a key innovation in an experimental Escherichia coli population. Nature 489, 513-518 (2012).

16. Fish, D. N., Piscitelli, S. C. \& Danziger, L. H. Development of resistance during antimicrobial therapy: a review of antibiotic classes and patient characteristics in 173 studies. Pharmacotherapy 15, 279-291 (1995).

17. Vogwill, T., Kojadinovic, M., Furio, V. \& MacLean, R. C. Testing the role of genetic background in parallel evolution using the comparative experimental evolution of antibiotic resistance. Mol. Biol. Evol. 31, 3314-3323 (2014).

18. Gordon, N. C. et al. Prediction of Staphylococcus aureus antimicrobial resistance by whole-genome sequencing. J. Clin. Microbiol. 52, 1182-1191 (2014).

19. Holden, M. T. et al. A genomic portrait of the emergence, evolution, and global spread of a methicillin-resistant Staphylococcus aureus pandemic. Genome Res. 23, 653-664 (2013).

20. Recker, M. et al. Clonal differences in Staphylococcus aureus bacteraemiaassociated mortality. Nat. Microbiol. 2, 1381-1388 (2017).

21. Drusano, G. L. Antimicrobial pharmacodynamics: critical interactions of 'bug and drug'. Nat. Rev. Microbiol. 2, 289-300 (2004).

22. Oliver, A., Canton, R., Campo, P., Baquero, F. \& Blazquez, J. High frequency of hypermutable Pseudomonas aeruginosa in cystic fibrosis lung infection. Science 288, 1251-1254 (2000).

23. Chewapreecha, C. et al. Comprehensive identification of single nucleotide polymorphisms associated with beta-lactam resistance within pneumococcal mosaic genes. PLoS Genet. 10, e1004547 (2014).
24. Laabei, M. et al. Predicting the virulence of MRSA from its genome sequence Genome Res. 24, 839-849 (2014).

25. Ferrero, L. et al. Cloning and primary structure of Staphylococcus aureus DNA topoisomerase IV: a primary target of fluoroquinolones. Mol. Microbiol. 13, 641-653 (1994)

26. Ito, H. et al. Quinolone resistance mutations in the DNA gyrase gyrA and gyrB genes of Staphylococcus aureus. Antimicrob. Agents Chemother. 38, 2014-2023 (1994)

27. Folkesson, A. et al. Adaptation of Pseudomonas aeruginosa to the cystic fibrosis airway: an evolutionary perspective. Nat. Rev. Microbiol. 10, 841-851 (2012).

28. Lieberman, T. D. et al. Parallel bacterial evolution within multiple patients identifies candidate pathogenicity genes. Nat. Genet. 43, 1275-1280 (2011).

29. Toprak, E. et al. Evolutionary paths to antibiotic resistance under dynamically sustained drug selection. Nat. Genet. 44, 101-105 (2011).

30. Vogwill, T., Phillips, R. L., Gifford, D. R. \& MacLean, R. C. Divergent evolution peaks under intermediate population bottlenecks during bacterial experimental evolution. Proc. Biol. Sci. 283, 20160749 (2016).

31. Hedge, J. \& Wilson, D. J. Practical approaches for detecting selection in microbial genomes. PLoS Comput. Biol. 12, e1004739 (2016).

32. Kaatz, G. W., Seo, S. M. \& Ruble, C. A. Efflux-mediated fluoroquinolone resistance in Staphylococcus aureus. Antimicrob. Agents Chemother. 37, 1086-1094 (1993).

33. Ubukata, K., Itoh-Yamashita, N. \& Konno, M. Cloning and expression of the norA gene for fluoroquinolone resistance in Staphylococcus aureus. Antimicrob. Agents Chemother. 33, 1535-1539 (1989)

34. Gallagher, L. A. et al. Tandem amplification of the staphylococcal cassette chromosome mec element can drive high-level methicillin resistance in methicillin-resistant Staphylococcus aureus. Antimicrobial Agents Chemother. 61, e00869-00817 (2017).

35. Hawkey, J. et al. ISMapper: identifying transposase insertion sites in bacterial genomes from short read sequence data. BMC Genomics 16, 667 (2015).

36. Sandegren, L. \& Andersson, D. I. Bacterial gene amplification: implications for the evolution of antibiotic resistance. Nat. Rev. Microbiol. 7, 578-588 (2009).

37. Andersson, D. I. \& Hughes, D. Gene amplification and adaptive evolution in bacteria. Annu. Rev. Genet. 43, 167-195 (2009).

38. Cirz, R. T. et al. Complete and SOS-mediated response of Staphylococcus aureus to the antibiotic ciprofloxacin. J. Bacteriol. 189, 531-539 (2007).

39. Torres-Barcelo, C., Kojadinovic, M., Moxon, R. \& MacLean, R. C. The SOS response increases bacterial fitness, but not evolvability, under a sublethal dose of antibiotic. Proc. Biol. Sci. 282, 20150885 (2015).

40. Monk, I. R., Shah, I. M., Xu, M., Tan, M. W. \& Foster, T. J. Transforming the untransformable: application of direct transformation to manipulate genetically Staphylococcus aureus and Staphylococcus epidermidis. mBio 3, e00277-00211-e00277-00211 (2012).

41. Neyfakh, A. A., Borsch, C. M. \& Kaatz, G. W. Fluoroquinolone resistance protein NorA of Staphylococcus aureus is a multidrug efflux transporter. Antimicrob. Agents Chemother. 37, 128-129 (1993).

42. Alexander, H. K., Martin, G., Martin, O. Y. \& Bonhoeffer, S. Evolutionary rescue: linking theory for conservation and medicine. Evol. Appl. 7, 1161-1179 (2014).

43. Martin, G., Aguilee, R., Ramsayer, J., Kaltz, O. \& Ronce, O. The probability of evolutionary rescue: towards a quantitative comparison between theory and evolution experiments. Philos. Trans. R. Soc. Lond. B Biol. Sci. 368, 20120088 (2013).

44. Alexander, H. K. \& MacLean, R. C. Stochastic bacterial population dynamics restrict the establishment of antibiotic resistance from single cells. Proc. Natl Acad. Sci. USA https://doi.org/10.1073/pnas.1919672117 (2020).

45. Costa, S. S. et al. Genetic diversity of norA, coding for a main efflux pump of Staphylococcus aureus. Front. Genet. 9, 710 (2019).

46. Kaatz, G. W., Thyagarajan, R. V. \& Seo, S. M. Effect of promoter region mutations and mgra overexpression on transcription of norA, which encodes a Staphylococcus aureus multidrug efflux transporter. Antimicrob. Agents Chemother. 49, 161-169 (2005)

47. Fournier, B., Truong-Bolduc, Q. C., Zhang, X. \& Hooper, D. C. A Mutation in the $5^{\prime}$ untranslated region increases stability of norA mRNA, encoding a multidrug resistance transporter of Staphylococcus aureus. J. Bacteriol. 183, 2367-2371 (2001)

48. Kaatz, G. W., Seo, S. M. \& Foster, T. J. Introduction of a norA promoter region mutation into the chromosome of a fluoroquinolone-susceptible strain of Staphylococcus aureus using plasmid integration. Antimicrob. Agents Chemother. 43, 2222-2224 (1999).

49. Truong-Bolduc, Q. C., Zhang, X. \& Hooper, D. C. Characterization of NorR protein, a multifunctional regulator of norA expression in Staphylococcus aureus. J. Bacteriol. 185, 3127-3138 (2003). 
50. Luong, T. T., Dunman, P. M., Murphy, E., Projan, S. J. \& Lee, C. Y. Transcription profiling of the mgrA regulon in Staphylococcus aureus. J. Bacteriol. 188, 1899-1910 (2006).

51. Truong-Bolduc, Q. C., Ding, Y. \& Hooper, D. C. Posttranslational modification influences the effects of MgrA on norA expression in Staphylococcus aureus. J. Bacteriol. 190, 7375-7381 (2008).

52. Jang, S. Multidrug efflux pumps in Staphylococcus aureus and their clinical implications. J. Microbiol. 54, 1-8 (2016).

53. Kaatz, G. W. \& Seo, S. M. Mechanisms of fluoroquinolone resistance in genetically related strains of Staphylococcus aureus. Antimicrob. Agents Chemother. 41, 2733-2737 (1997).

54. Marcusson, L. L., Frimodt-Moller, N. \& Hughes, D. Interplay in the selection of fluoroquinolone resistance and bacterial fitness. PLoS Pathog. 5, e1000541 (2009).

55. Oethinger, M., Kern, W. V., Jellen-Ritter, A. S., McMurry, L. M. \& Levy, S. B. Ineffectiveness of topoisomerase mutations in mediating clinically significant fluoroquinolone resistance in Escherichia coli in the absence of the AcrAB efflux pump. Antimicrob. Agents Chemother. 44, 10-13 (2000).

56. Bruchmann, S., Dötsch, A., Nouri, B., Chaberny, I. F. \& Häussler, S. Quantitative contributions of target alteration and decreased drug accumulation to Pseudomonas aeruginosa fluoroquinolone resistance. Antimicrob. Agents Chemother. 57, 1361-1368 (2013).

57. Lomovskaya, O. et al. Identification and characterization of inhibitors of multidrug resistance efflux pumps in Pseudomonas aeruginosa: novel agents for combination therapy. Antimicrob. Agents Chemother. 45, 105-116 (2001).

58. Farhat, M. R. et al. GWAS for quantitative resistance phenotypes in Mycobacterium tuberculosis reveals resistance genes and regulatory regions. Nat. Commun. 10, 1-11 (2019).

59. Everitt, R. G. et al. Mobile elements drive recombination hotspots in the core genome of Staphylococcus aureus. Nat. Commun. 5, 1-9 (2014).

60. Price, L. B. et al. Staphylococcus aureus CC398: host adaptation and emergence of methicillin resistance in livestock. mBio 3, 1-6 (2012).

61. Stegger, M. et al. Genome analysis of Staphylococcus aureus ST291, a double locus variant of ST398, reveals a distinct genetic lineage. PLOS ONE 8, e63008 (2013).

62. Mediavilla, J. R. et al. Methicillin-susceptible Staphylococcus aureus ST398, New York and New Jersey, USA. Emerg. Infect. Dis. 18, 700-702 (2012).

63. Mairi, A. et al. Distribution of toxinogenic methicillin-resistant and methicillin-susceptible Staphylococcus aureus from different ecological niches in Algeria. Toxins 11, 500 (2019).

64. Panahi, M. \& Saei, H. D. Genetic diversity and methicillin resistance of Staphylococcus aureus originating from buffaloes with mastitis in Iran. Comp. Immunol., Microbiol. Infect. Dis. 62, 19-24 (2019).

65. Knight, G. M., Budd, E. L. \& Lindsay, J. A. Large mobile genetic elements carrying resistance genes that do not confer a fitness burden in healthcareassociated meticillin-resistant Staphylococcus aureus. Microbiology 159, 1661-1672 (2013).

66. Lindsay, J. A. Staphylococcus aureus genomics and the impact of horizontal gene transfer. Int. J. Med. Microbiol. 304, 103-109 (2014).

67. Richardson, E. J. et al. Gene exchange drives the ecological success of a multihost bacterial pathogen. Nat. Ecol. Evol. 2, 1468-1478 (2018).

68. Didelot, X., Bowden, R., Wilson, D. J., Peto, T. E. A. \& Crook, D. W. Transforming clinical microbiology with bacterial genome sequencing. Nat. Rev. Genet. 13, 601-612 (2012).

69. Klemm, E. \& Dougan, G. Advances in understanding bacterial pathogenesis gained from whole-genome sequencing and phylogenetics. Cell Host Microbe 19, 599-610 (2016).

70. Earle, S. G. et al. Identifying lineage effects when controlling for population structure improves power in bacterial association studies. Nat. Microbiol. 1, 16041 (2016).

71. Brockhurst, M. A. et al. Assessing evolutionary risks of resistance for new antimicrobial therapies. Nat. Ecol. Evolution 3, 515-517 (2019).

72. El Meouche, I. \& Dunlop, M. J. Heterogeneity in efflux pump expression predisposes antibiotic-resistant cells to mutation. Science 362, 686-690 (2018).

73. Nolivos, S. et al. Role of AcrAB-TolC multidrug efflux pump in drug resistance acquisition by plasmid transfer. Science 364, 778-782 (2019).

74. Schindler, B. D., Jacinto, P. \& Kaatz, G. W. Inhibition of drug efflux pumps in Staphylococcus aureus: current status of potentiating existing antibiotics. Future Microbiol. 8, 491-507 (2013).

75. Ritz, C., Baty, F., Streibig, J. C. \& Gerhard, D. Dose-response analysis using R. PLOS ONE 10, e0146021 (2015).

76. Eyre, D. W. et al. A pilot study of rapid benchtop sequencing of Staphylococcus aureus and Clostridium difficile for outbreak detection and surveillance. BMJ Open 2, e001124 (2012).

77. Bolger, A. M., Lohse, M. \& Usadel, B. Trimmomatic: a flexible trimmer for Illumina sequence data. Bioinformatics 30, 2114-2120 (2014)
78. Lunter, G. \& Goodson, M. Stampy: a statistical algorithm for sensitive and fast mapping of Illumina sequence reads. Genome Res. 21, 936-939 (2011).

79. Li, H. et al. The sequence alignment/map format and SAMtools. Bioinformatics 25, 2078-2079 (2009).

80. Li, H. A statistical framework for SNP calling, mutation discovery, association mapping and population genetical parameter estimation from sequencing data. Bioinformatics 27, 2987-2993 (2011)

81. Altschul, S. F., Gish, W., Miller, W., Myers, E. W. \& Lipman, D. J. Basic local alignment search tool. J. Mol. Biol. 215, 403-410 (1990).

82. Bankevich, A. et al. SPAdes: a new genome assembly algorithm and its applications to single-cell sequencing. J. Comput. Biol. 19, 455-477 (2012).

83. Jolley, K. A., Bray, J. E. \& Maiden, M. C. J. Open-access bacterial population genomics: BIGSdb software, the PubMLST.org website and their applications. Wellcome Open Res. 3, 124 (2018).

84. Stamatakis, A. RAxML version 8: a tool for phylogenetic analysis and postanalysis of large phylogenies. Bioinformatics 30, 1312-1313 (2014).

85. Didelot, X. \& Wilson, D. J. ClonalFrameML: efficient inference of recombination in whole bacterial genomes. PLoS Comput. Biol. 11, e1004041 (2015).

86. Guindon, S. et al. New algorithms and methods to estimate maximumlikelihood phylogenies: assessing the performance of PhyML 3.0. Syst. Biol. 59, 307-321 (2010)

87. Seemann, T. Prokka: rapid prokaryotic genome annotation. Bioinformatics 30, 2068-2069 (2014)

88. Barrick, J. E. et al. Identifying structural variation in haploid microbial genomes from short-read resequencing data using breseq. BMC Genomics $\mathbf{1 5}$, 1039 (2014)

89. Li, H. \& Durbin, R. Fast and accurate short read alignment with BurrowsWheeler transform. Bioinformatics 25, 1754-1760 (2009).

90. Garrison, E. \& M. G. Haplotype-based variant detection from short-read sequencing. Preprint at http://arxiv.org/abs/1207.3907 (2012).

91. R: A Language and environment for statistical computing (R Foundation for Statistical Computing, Vienna, Austria, 2016)

92. Patro, R., Duggal, G., Love, M. I., Irizarry, R. A. \& Kingsford, C. Salmon provides fast and bias-aware quantification of transcript expression. Nat. Methods 14, 417-419 (2017).

93. Love, M. I., Huber, W. \& Anders, S. Moderated estimation of fold change and dispersion for RNA-seq data with DESeq2. Genome Biol. 15, 550 (2014).

94. Rueden, C. T. et al. ImageJ2: ImageJ for the next generation of scientific image data. BMC Bioinforma. 18, 529 (2017).

95. Zheng, Q. rSalvador: an R package for the fluctuation experiment. G3: Genes, Genomes, Genet. 7, 3849-3856 (2017).

96. Corrigan, R. M. \& Foster, T. J. An improved tetracycline-inducible expression vector for Staphylococcus aureus. Plasmid 61, 126-129 (2009).

\section{Acknowledgements}

This project was supported by Wellcome Trust Grant 106918/Z/15/Z. B.Y. is funded by a National Institute for Health Research (NIHR) Clinical Lectureship. We thank K. Foster, D. Wilson and A. San Millan for feedback. We thank the Oxford Genomics Centre at the Wellcome Centre for Human Genetics (funded by Wellcome Trust grant reference $203141 / \mathrm{Z} / 16 / \mathrm{Z}$ ) for the generation and initial processing of the sequencing data.

\section{Author contributions}

A.P., J.H. and R.C.M. conceived of the study and designed experiments. A.P., N.K. and B.Y. carried out experiments. J.H. and A.P. carried out bioinformatic work and data analysis. R.C.M. and A.P. wrote the paper, which was edited by all authors.

\section{Competing interests}

The authors declare no competing interests.

\section{Additional information}

Supplementary information is available for this paper at https://doi.org/10.1038/s41467 020-17735-y.

Correspondence and requests for materials should be addressed to A.P. or R.C.M.

Peer review information Nature Communications thanks the anonymous reviewers for their contribution to the peer review of this work. Peer reviewer reports are available.

Reprints and permission information is available at http://www.nature.com/reprints

Publisher's note Springer Nature remains neutral with regard to jurisdictional claims in published maps and institutional affiliations. 
(c) (i) Open Access This article is licensed under a Creative Commons Attribution 4.0 International License, which permits use, sharing, adaptation, distribution and reproduction in any medium or format, as long as you give appropriate credit to the original author(s) and the source, provide a link to the Creative Commons license, and indicate if changes were made. The images or other third party material in this article are included in the article's Creative Commons license, unless indicated otherwise in a credit line to the material. If material is not included in the article's Creative Commons license and your intended use is not permitted by statutory regulation or exceeds the permitted use, you will need to obtain permission directly from the copyright holder. To view a copy of this license, visit http://creativecommons.org/ licenses/by/4.0/.

(C) The Author(s) 2020 